\title{
含有对氨基苯甲酸和苯磺酰胺结构单元的新型分子及其抗糖尿病活性
}

\author{
杨 龙 ${ }^{a}$ 晏菊芳 ${ }^{b}$ 范 莉 ${ }^{a}$ 陈 欣 ${ }^{b}$ 上官瑞燕 ${ }^{a}$ \\ 汪林发 ${ }^{a}$ 杨大成*,a \\ ( ${ }^{a}$ 西南大学化学化工学院 重庆 400715) \\ ${ }^{b}$ 成都地奥制药集团药物篮选中心 成都 610041)
}

\begin{abstract}
摘要 基于本实验室前期发现的高活性分子的结构特征，作者设计了含有对氨基苯甲酸和苯磺酰胺结构单元的新型分 子. 通过 4 条合成路线的探索, 发现了中间体 IM1 IM3 及目标分子 TM1 和 TM2 的简捷合成路线和实用合成方法; 采 用本实验室前期建立的合成方法，顺利得到设计的 26 个化合物，合成方法简便，反应条件温和，收率为 $64 \% \sim 95 \% .21$ 个新化合物通过 ${ }^{1} \mathrm{H} N M R,{ }^{13} \mathrm{C}$ NMR 和 HRMS 进行结构表征. 目前的体外抗糖尿病活性结果显示, 所得 26 个分子的过 氧化物酶体增殖物激活受体(PPAR)的激动活性较弱. 本研究进一步证实了醇/二氯亚砜体系脱除脂肪酰芳胺酰基的能 力，有助于含有对氨基苯甲酸和苯磺酰胺结构单元分子的进一步结构优化.
\end{abstract}

关键词＼cjkstart糖尿病；对氨基苯甲酸；磺胺；过氧化物酶体增殖物激活受体；合成; 活性

\section{Synthesis and Antidiabetic Activity of Novel Molecules Containing p-Aminobenzoic Acid and Benzenesulfonamide Moiety}

\author{
Yang, Long ${ }^{a} \quad$ Yan, Jufang ${ }^{b} \quad{\text { Fan, } \mathrm{Li}^{a} \quad \text { Chen, } \text { Xin }^{b} \quad \text { Shangguan, Ruiyan }}^{a}$ \\ Wang, Linfa ${ }^{a} \quad$ Yang, Dacheng ${ }^{*, a}$ \\ $\left({ }^{a}\right.$ School of Chemistry and Chemical Engineering, Southwest University, Chongqing 400715) \\ ( ${ }^{b}$ Drug Screening Center, Chengdu Di'Ao Pharmaceutical Group Co., Ltd., Chengdu 610041)
}

\begin{abstract}
A new class of target molecules containing $p$-aminobenzoic acid and benzenesulfonamide moiety is reported, based on the structural features of a series of analogues with strong biological activities previously synthesized by the group. By taking 4 discrete synthetic routes, the practical procedures and facile preparative routes for both the intermediates of IM1 IM3 and the target molecules of TM1 and TM2 were established. A total of 26 designed compounds were synthesized smoothly using the established synthetic approaches under mild reaction conditions, with low cost and high yields $(64 \% \sim$ $95 \%$ ). The chemical structures of 21 new compounds were confirmed by ${ }^{1} \mathrm{H}$ NMR, ${ }^{13} \mathrm{C}$ NMR and HRMS techniques. The bioassay test demonstrates weak antidiabetic activity for all the target molecules. This study has further expanded the application of alcohol/ $\mathrm{SOCl}_{2}$ system in the deacylation of $N$-arylacetamides and chloro- $N$-arylacetamides as well as esterification of carboxy group concomitantly, which is supportive to the structure optimization of novel molecules containing $p$-aminobenzoic acid and benzenesulfonamide moiety.
\end{abstract}

Keywords diabetes mellitus; $p$-aminobenzoic acid; sulfanilamide; peroxisome proliferator-activated receptors (PPAR); synthesis; activity

糖尿病是人体内糖、蛋白质、脂肪代谢紊乱造成的 一种慢性进行性疾病, 现已成为世界上继肿瘤、心脑血 管疾病之后第三大严重危害人类身体健康的慢性非传 染性疾病. 糖尿病全球发病率为 3\% 10\%, 其中 95\%以 上是 2 型糖尿病 $(\mathrm{T} 2 \mathrm{DM})$. 用于治疗 $\mathrm{T} 2 \mathrm{DM}$ 的常见药物
很多，诸如胰岛素分泌促进剂、胰岛素增敏剂 ${ }^{[1]} 、 \alpha$-葡 萄糖苷酶抑制剂 ${ }^{[2]}$ 等. 这些药物种类众多, 作用靶点各 异, 治疗效果显著, 为世界范围糖尿病的治疗做出了重 要贡献. 但是, 有的药物原发效应较差; 有的可能加重 心力衰竭患者的病情，有使心血管不良事件和充血性心

*E-mail: hxydc@swu.edu.cn

Received April 13, 2012; revised May 31, 2012; published online June 6, 2012.

Project supported by the Scientific and Technological Project in Chongqing City (Nos. 2011AB5001, 2011AC1053, 2011AC5107).

重庆市科技攻关计划(Nos. 2011AB5001, 2011AC1053, 2011AC5107)资助项目. 
力衰竭发生率增加的风险; 有的药物存在腹泻、胃肠胀 气、轻微腹痛等不良反应 ${ }^{[3]}$. 因此, 设计和开发新型的 T2DM 治疗药物, 克服现有药物程度不同的副作用, 仍 然是 T2DM 治疗药物研究的难点和热点.

选择活性片段构建新型目标分子是目前认可的药 物设计方法. 众所周知, 磺胺药物的发现是抗菌药物史 上的重大事件, 苯磺酰胺类抗菌药物是目前常用抗菌药 物 ${ }^{[4]}$; 某些苯磺酰胺类化合物还具有利尿 ${ }^{[5]}$ 、抗甲状

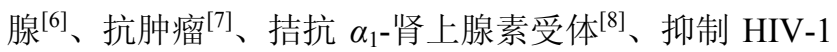
蛋白酶 ${ }^{[9]}$ 以及降血糖 ${ }^{[10]}$ 等生物活性. 文献调研发现, 对 氨基苯甲酸(PABA)参与初级代谢中叶酸的合成 ${ }^{[11]}$; 此 外, PABA 的氨基酸衍生物已被用于胸苷酸合成酶 ${ }^{[12]}$ 、 二氢叶酸还原酶 ${ }^{[13]}$ 、法尼基转移酶 ${ }^{[14]}$ 等重要抑制剂的合 成; 含有 PABA 结构单元的某些衍生物具有抗肿瘤 ${ }^{[15]}$ 、 抗癌 ${ }^{[16]}$ 、抗乙型肝炎病毒 ${ }^{[17]} 、$ 抗 HIV $^{[18]}$ 、抗菌 ${ }^{[19]}$ 、降

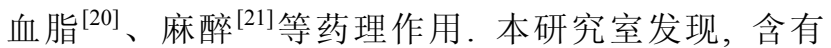
PABA 或苯磺酰胺结构单元的某些 $\beta$-氨基酮(醇)具有很 好的过氧化物酶体增殖物激活受体(peroxisome proliferator-activated receptors, PPAR) 激动活性 ${ }^{[22 \sim 25]}$ (参见
Chart 1).

这些研究结果启示我们, PABA 或苯磺酰胺可作为 具有抗糖尿病活性分子的活性片段. 据此设想, 将 PABA 和苯磺酰胺结构单元同时引入新分子结构中, 以 活性片段的拼合设计新型分子(Scheme 1), 希望获取具 有抗糖尿病活性的新化合物.

分析 Chart 1 的分子结构发现, PABA 片段羧基裸露 但氨基修饰, 分子可能具有较好活性, 如 1 和 2 ; 进一 步, 类似分子的 PABA 片段羧基简单酯化, 所得分子(如 3)仍有令人惊喜的活性. 但是, 仅将 PABA 右端羧基以 氨基酸片段衍生, 所得分子仅有较弱的抗糖尿病活 性 ${ }^{[26]}$. 由此我们认为, PABA 氨基端的修饰是必需的, 而羧基端采用太大体积的基团衍生是不利的，可能羧基 端空间体积太大会阻碍其与配体的结合. 所以，作者决 定将 PABA 羧基端用小基团(简单烷基)修饰、氨基端用 空间体积较大的苯磺酰胺结构单元衍生(Scheme 1).

4 进一步改变为 5 和 6 , 所得分子活性更好, 表明苯 磺酰胺结构单元的左右两端同时修饰也是可行的, 亦即 上述模型分子磺酰胺的氨基端还可以进一步修饰. 作为<smiles>COc1ccc2cc(CCC(O)CC(Nc3ccc(C(=O)O)cc3)c3cccc([N+](=O)[O-])c3)ccc2c1</smiles>

PPAR relative activation $76.21 \%$

PPAR relative activation $87.02 \%$

PPAR relative activation $130.91 \%$<smiles>C=NC(=N)NS(=O)(=O)c1ccc(NC(CC(=O)c2ccc(Br)cc2)c2ccc(Cl)c(Cl)c2)cc1</smiles>

PPAR relative activation $86.90 \%$

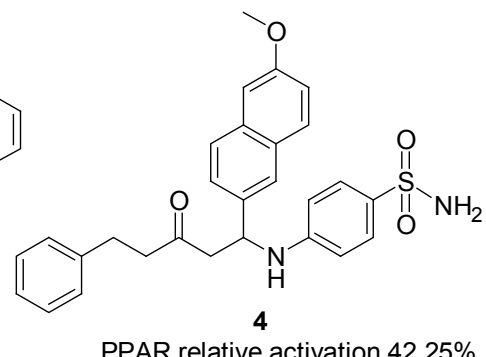

PPAR relative activation $42.25 \%$<smiles>COc1ccc2cc(CCC(=O)CC(Nc3ccc(S(=O)(=O)Nc4cc(C)on4)cc3)c3ccc4c(c3)OCO4)ccc2c1</smiles>

PPAR relative activation $135.47 \%$

\section{Chart 1}<smiles>[R]OC(=O)c1ccc(NC(=O)Nc2ccc(S(=O)(=O)Nc3ccc(C(=O)O[R])cc3)cc2)cc1</smiles>

Scheme 1 
本研究的一种尝试, 我们认为将苯磺酰胺左端氨基以各 种酰基结构单元进一步衍生，可能会得到抗糖尿病活性 分子. 据此, 目标分子的结构演变为如 TM1 和 TM2 所 示模式(Scheme 1).

目标分子的合成路线很多, 见 Scheme 2. 本研究得 到 26 个化合物, 21 个为新化合物.

\section{1 实验部分}

\section{1 主要检测仪器和试剂}

AV-300 型超导核磁共振仪(Bruker, USA; $300 \mathrm{MHz}$, TMS 为内标); HR ESI-FTICR MS (Varian 7. OT, USA); X-6 型精密显微熔点测定仪(北京福凯仪器有限公司). 对氨基苯甲酸(爱斯特(成都)医药技术有限公司, AR), 对乙酰氨基苯磺酰氯(上海达瑞精细化学品有限公司, $\mathrm{CP})$, 其它各种试剂均为化学纯或分析纯.

\section{2 中间体和目标分子的合成}

\subsubsection{4-(4-乙酰胺基苯磺酰胺基)苯甲酸(IM1)的制} 备

$100 \mathrm{~mL}$ 圆底烧瓶中加入 $\mathrm{K}_{2} \mathrm{CO}_{3} 28.9 \mathrm{~g}(210 \mathrm{mmol})$ 和水 $8 \mathrm{~mL}$, 溶解后冰浴冷却, 搅拌下加入对氨基苯甲酸 $13.7 \mathrm{~g}$ (100 mmol). 溶解冷却后, 缓慢滴加对乙酰胺基 苯磺酰氯 $35.2 \mathrm{~g}$ (151 mmol) 的丙酩 $(70 \mathrm{~mL})$ 溶液, 加毕继 续摚拌 $7 \mathrm{~min}$, 撤去冰水浴, 室温搅拌反应, TLC 监测反 应进程. 反应完毕, 减压蒸馏除去丙酮, 加水 $40 \mathrm{~mL}, 2$ $\mathrm{mol} \cdot \mathrm{L}^{-1} \mathrm{HCl}$ 水溶液调节 $\mathrm{pH}=1 \sim 2$, 析出大量固体. 抽 滤, 纯水 $(20 \mathrm{~mL} \times 3)$ 洗涤滤饼. 抽干, 滤饼干燥, 即得 4-(4-乙酰胺基苯磺酰胺基)苯甲酸(IM1) ${ }^{[27]}$.

\section{2 .24 -(4-氨基苯磺酰胺基)苯甲酸(IM2)的制备}

$100 \mathrm{~mL}$ 圆底烧瓶中加入 IM1 $8.8 \mathrm{~g}$ (26.4 mmol)和 2 $\mathrm{mol} \cdot \mathrm{L}^{-1} \mathrm{KOH}$ 溶液 $50 \mathrm{~mL}, 50{ }^{\circ} \mathrm{C}$ 搅拌反应, $\mathrm{TLC}$ 监测反 应进程. 反应完毕后, $2 \mathrm{~mol} \cdot \mathrm{L}^{-1} \mathrm{HCl}$ 水溶液调节至 $\mathrm{pH}=$ 3 , 析出大量黄白色固体, 抽滤, 纯水 $(15 \mathrm{~mL} \times 3)$ 洗涤滤 饼. 滤饼干燥, 得 4-(4-氨基苯磺酰胺基)苯甲酸(IM2) $)^{[27]}$. 1.2 .3 4-(4-酰胺基苯磺酰胺基)苯甲酸(TM1)的制备

$100 \mathrm{~mL}$ 圆底烧瓶中加入 IM2 $2.9 \mathrm{~g}$ (10.0 mmol)和 丙酮 $10 \mathrm{~mL}$, 充分搅拌使其溶解; 加入 $\mathrm{K}_{2} \mathrm{CO}_{3} 4.2 \mathrm{~g}(30.2$ mmol), $15 \sim 19{ }^{\circ} \mathrm{C}$ 搅拌 $30 \mathrm{~min}$, 冰浴冷却, 缓慢滴加酰 氯 $14.9 \mathrm{mmol}$ 的丙酩 $(10 \mathrm{~mL})$ 溶液. 加毕, 继续搅拌 30 $\min$, 撤去冰水浴, 室温搅拌反应, TLC 监测反应进程. 反应完毕 $(10 \sim 17 \mathrm{~h})$ 后, 减压蒸馏除去丙酮, 加入饱和 食盐水 $20 \mathrm{~mL}, 2 \mathrm{~mol} \cdot \mathrm{L}^{-1} \mathrm{HCl}$ 水溶液调节 $\mathrm{pH}=2$, 析出 固体. 静置, 抽滤, 滤饼干燥, 得到产品 TM1a TM1d.

\subsubsection{4-(4-氨基苯磺酰胺基)苯甲酸酯(IM3)的合成}

路线 B: $100 \mathrm{~mL}$ 圆底烧瓶中加入醇 $15 \mathrm{~mL}$, 冰浴冷 却下缓慢滴加 $\mathrm{SOCl}_{2} 12.8 \mathrm{mmol}$, 加毕摚拌 $30 \mathrm{~min}$, 再加 入 IM1 3. 3 g (10 mmol, 表 2, Entry 2), 继续搅拌 $15 \mathrm{~min}$ 后转入油浴装置, 加热回流, TLC 监测反应进程. 反应

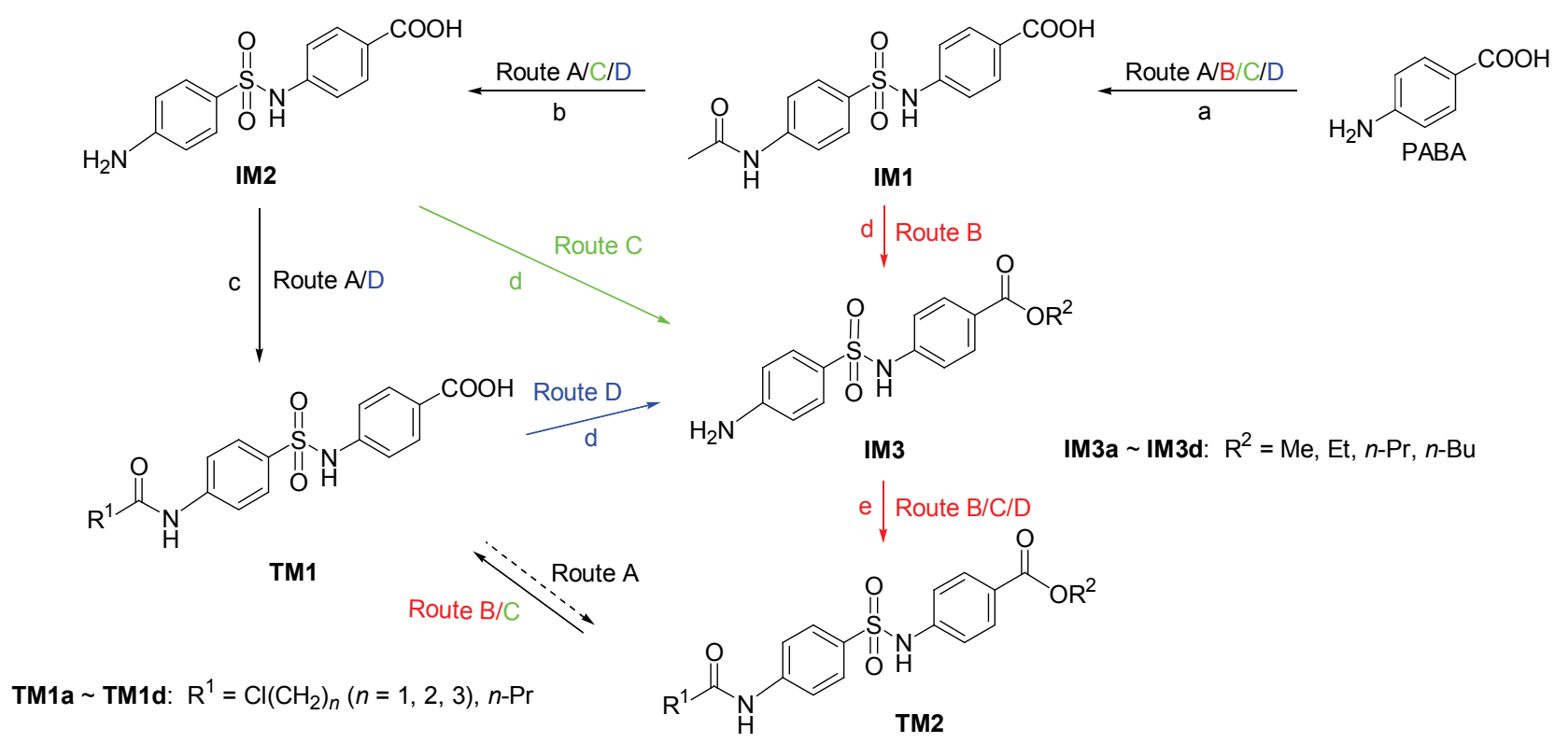

Reagents and conditions: (a) ASC, $\mathrm{K}_{2} \mathrm{CO}_{3}, \mathrm{Me}_{2} \mathrm{CO}$, r.t.; (b) $\mathrm{KOH}, \mathrm{H}_{2} \mathrm{O}, 50{ }^{\circ} \mathrm{C}$; (c) $\mathrm{R}^{1} \mathrm{COCl}, \mathrm{K}_{2} \mathrm{CO}_{3}$, r.t.; (d) $\mathrm{R}^{2} \mathrm{OH} / \mathrm{SOCl}_{2}$, reflux; (e) $\mathrm{R}^{1} \mathrm{COCl}$, $\mathrm{K}_{2} \mathrm{CO}_{3}$, THF

Scheme 2 
完毕后, 减压蒸馏除去溶剂, 加水 $30 \mathrm{~mL}$ 和乙酸乙酯 20 $\mathrm{mL}$ 混匀, 饱和碳酸钾溶液调节 $\mathrm{pH}=7 \sim 8$, 静置分层. 分液, 水层用乙酸乙酯 $(20 \mathrm{~mL} \times 2)$ 萃取, 合并有机层, 无水 $\mathrm{MgSO}_{4}$ 干燥, 抽滤, 滤液减压蒸馏除去溶剂, 乙醚/ 石油醚(体积比为 $1: 3$ )分散, 抽滤, 滤饼干燥, 得到 IM3 纯品. 表 2, Entries 7, 12, 18 实验与此类似.

路线 C: 路线 B 中的 IM1 以 IM2 $0.6 \mathrm{~g}(2 \mathrm{mmol})$ 替 换(表 2, Entry 17), 操作与路线 B 相同. 表 2, Entry 1 实 验与此类似.

路线 D: $100 \mathrm{~mL}$ 圆底烧瓶中加入醇 $8 \mathrm{~mL}$, 冰浴冷却 下缓慢滴加 $\mathrm{SOCl}_{2} 1.5 \mathrm{mmol}$, 加毕摚拌 $30 \mathrm{~min}$, 再加入 TM1 (1 mmol, 表 2, Entry 3), 继续搅拌 $15 \mathrm{~min}$ 后加热回 流, TLC 监测反应进程. 反应完毕后, 减压蒸馏除去溶 剂, 再加入水 $25 \mathrm{~mL}$ 和乙酸乙酯 $25 \mathrm{~mL}$ 混匀, 用饱和碳 酸钾溶液调节 $\mathrm{pH}$ 至 $7 \sim 8$, 静置分层. 分液, 乙酸乙酯 $(20 \mathrm{~mL} \times 2)$ 萃取水层, 合并有机层, 无水 $\mathrm{MgSO}_{4}$ 干燥, 抽滤, 滤液减压蒸馏除去溶剂, 乙酸乙酯/石油醚(体积 比为 $1: 3$ )分散, 抽滤, 滤饼干燥, 得到产品. 表 2 , Entries $4 \sim 6,8 \sim 11,13 \sim 16,19 \sim 22$ 实验与此类似.

\subsubsection{4-[4-酰胺基苯磺酰胺基]苯甲酸酯(TM2)的制} 备

$100 \mathrm{~mL}$ 圆底烧瓶中加入 IM3 $1.1 \mathrm{mmol}$ 和 THF 5 $\mathrm{mL}$, 摚拌溶解后加入 $\mathrm{K}_{2} \mathrm{CO}_{3} 0.2 \mathrm{~g}(1.5 \mathrm{mmol})$, 室温摚拌 $30 \mathrm{~min}$, 缓慢滴加酰氯 $1.3 \mathrm{mmol}$ 的 THF (3 mL)溶液, 室 温摚拌反应, TLC 监测反应过程. 反应完毕后, 减压蒸 馏除去溶剂, 加水 $25 \mathrm{~mL}$ 和乙酸乙酯 $25 \mathrm{~mL}$ 混匀, 2 $\mathrm{mol} \cdot \mathrm{L}^{-1} \mathrm{HCl}$ 水溶液调节 $\mathrm{pH}=3 \sim 4$, 静置分层. 分液, 乙酸乙酯 $(10 \mathrm{~mL})$ 萃取水层. 合并有机层, 加入饱和食盐 水 $25 \mathrm{~mL}$, 静置分层, 收集有机相. 无水 $\mathrm{MgSO}_{4}$ 干燥, 抽滤, 滤液减压蒸馏除去溶剂, 乙酸乙酯/石油醚(体积 比为 $1:$ 1)分散, 抽滤, 滤饼干燥, 得到产品 TM2a TM2p. 其他实验与此类似.

\subsection{PPRE-Luc 报告基因篮选实验}

HepG2 细胞接种于 96 孔板, 培养过夜后换用含待 测样品的低糖 DMEM 培养基, 同时设立空白对照(不加 样品)和阳性对照(加入阳性参照物吡格列酮). 继续培养 $24 \mathrm{~h}$ 后检测苂光素酶活性. 根据检测到的化学发光强度 $\mathrm{L}$ 值计算激动率, 样品的绝对激动率 $=(L$ 样品 $L$ 空白 1) $\times 100 \%$, 样品的相对激动率=样品的绝对激动率/阳 性样品的绝对激动率 $\times 100 \%$. 初篎时每个样品单个浓 度设双复孔, 重复两次.

\section{4 化合物数据及表征}

4-(4-乙酰胺基苯磺酰胺基)苯甲酸(IM1): 黄色固 体, 收率 $91 \% \sim 93 \%$. m.p. $244.1 \sim 246.5{ }^{\circ} \mathrm{C}$ (文献值 ${ }^{[27]}$
$251 \sim 255{ }^{\circ} \mathrm{C}$ ); ${ }^{1} \mathrm{H}$ NMR (DMSO- $\left.d_{6}, 300 \mathrm{MHz}\right) \delta: 12.72$ (s, $1 \mathrm{H}, \mathrm{COOH}), 10.74\left(\mathrm{~s}, 1 \mathrm{H}, \mathrm{SO}_{2} \mathrm{NH}\right), 10.38(\mathrm{~s}, 1 \mathrm{H}$, $\mathrm{CONH}), 7.80(\mathrm{~d}, J=9.0 \mathrm{~Hz}, 2 \mathrm{H}, \mathrm{ArH}), 7.71 \sim 7.75(\mathrm{~m}, 4 \mathrm{H}$, $\mathrm{ArH}), 7.18$ (d, J=8.4 Hz, 2H, $\mathrm{ArH}$ ), $2.06\left(\mathrm{~s}, 3 \mathrm{H}, \mathrm{CH}_{3}\right)$.

4-(4-氨基苯磺酰胺基)苯甲酸(IM2): 黄色固体, 收 率 94.7\%. m.p. 203.5 205.4 ${ }^{\circ} \mathrm{C}$ (文献值 ${ }^{[27]} 203 \sim 205$ ${ }^{\circ} \mathrm{C}$ ); ${ }^{1} \mathrm{H}$ NMR (DMSO- $\left.d_{6}, 300 \mathrm{MHz}\right) \delta$ : $10.39(\mathrm{~s}, 1 \mathrm{H}$, $\mathrm{COOH}), 7.78$ (d, $J=8.0 \mathrm{~Hz}, 2 \mathrm{H}, \mathrm{ArH}), 7.45$ (d, $J=8.0 \mathrm{~Hz}$, $2 \mathrm{H}, \mathrm{ArH}), 7.15$ (d, $J=8.0 \mathrm{~Hz}, 2 \mathrm{H}, \mathrm{ArH}), 6.55$ (d, $J=8.4$ $\mathrm{Hz}, 2 \mathrm{H}, \mathrm{ArH}), 6.03$ (s, 2H, $\mathrm{NH}_{2}$ ).

4-[4-(2-氯乙酰胺基)苯磺酰胺基]苯甲酸(TM1a): 白色固体, 收率 92.3\%. m.p. 230.7 232.6 ${ }^{\circ} \mathrm{C} ;{ }^{1} \mathrm{H}$ NMR (DMSO- $\left.d_{6}, 300 \mathrm{MHz}\right) \delta: 12.68(\mathrm{~s}, 1 \mathrm{H}, \mathrm{COOH}), 10.73(\mathrm{~s}$, $\left.1 \mathrm{H}, \mathrm{SO}_{2} \mathrm{NH}\right), 10.67(\mathrm{~s}, 1 \mathrm{H}, \mathrm{CONH}), 7.81 \sim 7.72(\mathrm{~m}, 6 \mathrm{H}$, $\mathrm{ArH}), 7.18(\mathrm{~d}, J=8.4 \mathrm{~Hz}, 2 \mathrm{H}, \mathrm{ArH}), 4.27\left(\mathrm{~s}, 2 \mathrm{H}, \mathrm{CH}_{2}\right) ;{ }^{13} \mathrm{C}$ NMR (DMSO- $\left.d_{6}, 75 \mathrm{MHz}\right) \delta$ : $166.81,165.41,142.59$, $142.03,133.58,130.76(2 \times \mathrm{C}), 128.17(2 \times \mathrm{C}), 125.53$, $119.23(2 \times \mathrm{C}), 118.13(2 \times \mathrm{C}), 43.52$; HRMS calcd for $\mathrm{C}_{15} \mathrm{H}_{12} \mathrm{ClN}_{2} \mathrm{O}_{5} \mathrm{~S}([\mathrm{M}-\mathrm{H}])^{-}$367.0161, found 367.0164.

4-[4-(3-氯丙酰胺基)苯磺酰胺基]苯甲酸(TM1b): 白色固体, 收率 94.0\%. m.p. 181.7 182.3 ${ }^{\circ} \mathrm{C} ;{ }^{1} \mathrm{H}$ NMR (DMSO- $\left.d_{6}, 300 \mathrm{MHz}\right) \delta: 12.61(\mathrm{~s}, 1 \mathrm{H}, \mathrm{COOH}), 10.72(\mathrm{~s}$, $\left.1 \mathrm{H}, \mathrm{SO}_{2} \mathrm{NH}\right), 10.46(\mathrm{~s}, 1 \mathrm{H}, \mathrm{CONH}), 7.81 \sim 7.73(\mathrm{~m}, 6 \mathrm{H}$, ArH), 7.18 (d, $J=7.8 \mathrm{~Hz}, 2 \mathrm{H}, \operatorname{ArH}), 3.86(\mathrm{t}, J=6.0 \mathrm{~Hz}$, $\left.2 \mathrm{H}, \mathrm{CH}_{2}\right), 2.84\left(\mathrm{t}, J=6.0 \mathrm{~Hz}, 2 \mathrm{H}, \mathrm{CH}_{2}\right) ;{ }^{13} \mathrm{C} \mathrm{NMR}$ (DMSO- $\left.d_{6}, 75 \mathrm{MHz}\right) \delta: 168.80,166.78,143.04,142.09$, $133.03,130.76(2 \times \mathrm{C}), 128.13(2 \times \mathrm{C}), 125.48,118.88$ $(2 \times \mathrm{C}), 118.07(2 \times \mathrm{C}), 40.53,39.32$; HRMS calcd for $\mathrm{C}_{16} \mathrm{H}_{14} \mathrm{ClN}_{2} \mathrm{O}_{5} \mathrm{~S}([\mathrm{M}-\mathrm{H}])^{-} \quad 381.0317$, found 381.0310.

4-[4-(4-氯丁酰胺基)苯磺酰胺基]苯甲酸(TM1c): 灰白色固体, 收率 95.1\%. m.p. 186.7 187.6 ${ }^{\circ} \mathrm{C} ;{ }^{1} \mathrm{H}$ NMR (DMSO- $\left.d_{6}, 300 \mathrm{MHz}\right) \delta: 10.72\left(\mathrm{~s}, 1 \mathrm{H}, \mathrm{SO}_{2} \mathrm{NH}\right)$, 10.43 (s, 1H, CONH), $7.81 \sim 7.75(\mathrm{~m}, 6 \mathrm{H}, \mathrm{ArH}), 7.19(\mathrm{~d}$, $J=8.4 \mathrm{~Hz}, 2 \mathrm{H}, \mathrm{ArH}), 3.68\left(\mathrm{t}, J=6.3 \mathrm{~Hz}, 2 \mathrm{H}, \mathrm{CH}_{2}\right), 2.51 \sim$ $2.47\left(\mathrm{~m}, 2 \mathrm{H}, \mathrm{CH}_{2}\right), 2.06 \sim 1.97\left(\mathrm{~m}, 2 \mathrm{H}, \mathrm{CH}_{2}\right) ;{ }^{13} \mathrm{C} \mathrm{NMR}$ (DMSO- $\left.d_{6}, 75 \mathrm{MHz}\right) \delta$ : 171.10, 166.88, 143.37, 142.17, $132.72,130.74(2 \times \mathrm{C}), 128.07(2 \times \mathrm{C}), 125.45,118.79$ $(2 \times \mathrm{C}), 118.08(2 \times \mathrm{C}), 44.95,33.38,27.67 \mathrm{~cm}^{-1}$; HRMS calcd for $\mathrm{C}_{17} \mathrm{H}_{16} \mathrm{ClN}_{2} \mathrm{O}_{5} \mathrm{~S}([\mathrm{M}-\mathrm{H}])^{-}$395.0474, found 395.0475 .

4-(4-丁酰氨基苯磺酰氨基)苯甲酸(TM1d): 白色固 体, 收率 89.0\%. m.p. 226.3 227.6 ${ }^{\circ} \mathrm{C}$ (文献值 ${ }^{[28]} 226 \sim$ $\left.228{ }^{\circ} \mathrm{C}\right) ;{ }^{1} \mathrm{H}$ NMR (DMSO- $\left.d_{6}, 300 \mathrm{MHz}\right) \delta: 12.43(\mathrm{~s}, 1 \mathrm{H}$, $\mathrm{COOH}), 10.69\left(\mathrm{~s}, 1 \mathrm{H}, \mathrm{SO}_{2} \mathrm{NH}\right), 10.25(\mathrm{~s}, 1 \mathrm{H}, \mathrm{CONH})$, 7.79 (d, $J=8.4 \mathrm{~Hz}, 2 \mathrm{H}, \mathrm{ArH}), 7.75$ (s, 4H, ArH), 7.18 (d, 
$J=8.7 \mathrm{~Hz}, 2 \mathrm{H}, \mathrm{ArH}), 2.29\left(\mathrm{t}, J=6.9 \mathrm{~Hz}, 2 \mathrm{H}, \mathrm{CH}_{2}\right), 1.65 \sim$ $1.52\left(\mathrm{~m}, 2 \mathrm{H}, \mathrm{CH}_{2}\right), 0.89\left(\mathrm{t}, J=7.2 \mathrm{~Hz}, 3 \mathrm{H}, \mathrm{CH}_{3}\right)$.

4-(4-氨基苯磺酰胺基)苯甲酸甲酯(IM3a): 黄色固 体, 收率 97.1\%. m.p. $238.7 \sim 239.5{ }^{\circ} \mathrm{C}$ (文献值 ${ }^{[29]}$ $248.2 \sim 250.7{ }^{\circ} \mathrm{C}$ ); ${ }^{1} \mathrm{H}$ NMR (DMSO- $\left.d_{6}, 300 \mathrm{MHz}\right) \delta$ : $10.46\left(\mathrm{~s}, 1 \mathrm{H}, \mathrm{SO}_{2} \mathrm{NH}\right), 7.80$ (d, J=9.0 Hz, 2H, ArH), 7.45 (d, $J=8.7 \mathrm{~Hz}, 2 \mathrm{H}, \mathrm{ArH}), 7.17(\mathrm{~d}, J=9.0 \mathrm{~Hz}, 2 \mathrm{H}, \mathrm{ArH})$, $6.54(\mathrm{~d}, J=8.7 \mathrm{~Hz}, 2 \mathrm{H}, \mathrm{ArH}), 6.05\left(\mathrm{~s}, 2 \mathrm{H}, \mathrm{NH}_{2}\right), 3.78$ (s, $\left.3 \mathrm{H}, \mathrm{CH}_{3}\right)$.

4-(4-氨基苯磺酰胺基)苯甲酸乙酯(IM3b): 黄色固 体, 收率 94.8\%. m.p. $233.7 \sim 235.1{ }^{\circ} \mathrm{C}$ (文献值 ${ }^{[29]}$ $247.1 \sim 249.3{ }^{\circ} \mathrm{C}$ ); ${ }^{1} \mathrm{H}$ NMR (DMSO- $\left.d_{6}, 300 \mathrm{MHz}\right) \delta$ : 10.48 (s, $\left.1 \mathrm{H}, \mathrm{SO}_{2} \mathrm{NH}\right), 7.80$ (d, $\left.J=8.7 \mathrm{~Hz}, 2 \mathrm{H}, \mathrm{ArH}\right), 7.46$ (d, $J=8.7 \mathrm{~Hz}, 2 \mathrm{H}, \mathrm{ArH}), 7.18(\mathrm{~d}, J=8.7 \mathrm{~Hz}, 2 \mathrm{H}, \mathrm{ArH})$, $6.54(\mathrm{~d}, J=8.7 \mathrm{~Hz}, 2 \mathrm{H}, \mathrm{ArH}), 6.07\left(\mathrm{~s}, 2 \mathrm{H}, \mathrm{NH}_{2}\right), 4.28 \sim$ $4.21\left(\mathrm{~m}, 2 \mathrm{H}, \mathrm{CH}_{2}\right), 1.27\left(\mathrm{t}, J=7.2 \mathrm{~Hz}, 3 \mathrm{H}, \mathrm{CH}_{3}\right)$.

4-(4-氨基苯磺酰胺基)苯甲酸丙酯(IM3c): 黄色固 体, 收率 93.7\%. m.p. 225.3 227.0 ${ }^{\circ} \mathrm{C} ;{ }^{1} \mathrm{H}$ NMR (DMSO- $\left.d_{6}, 300 \mathrm{MHz}\right) \delta: 10.48\left(\mathrm{~s}, 1 \mathrm{H}, \mathrm{SO}_{2} \mathrm{NH}\right), 7.81(\mathrm{~d}$, $J=8.7 \mathrm{~Hz}, 2 \mathrm{H}, \mathrm{ArH}), 7.46$ (d, $J=9.0 \mathrm{~Hz}, 2 \mathrm{H}, \mathrm{ArH}), 7.18$ (d, $J=8.7 \mathrm{~Hz}, 2 \mathrm{H}, \mathrm{ArH}), 6.55(\mathrm{~d}, J=8.7 \mathrm{~Hz}, 2 \mathrm{H}, \mathrm{ArH})$, 6.07 (s, $\left.2 \mathrm{H}, \mathrm{NH}_{2}\right), 4.15$ (t, $\left.J=6.6 \mathrm{~Hz}, 2 \mathrm{H}, \mathrm{CH}_{2}\right), 1.73 \sim$ $1.61\left(\mathrm{~m}, 2 \mathrm{H}, \mathrm{CH}_{2}\right), 0.93\left(\mathrm{t}, J=7.5 \mathrm{~Hz}, 3 \mathrm{H}, \mathrm{CH}_{3}\right)$.

4-(4-氨基苯磺酰胺基)苯甲酸丁酯(IM3d): 黄色固 体, 收率 93.3\%. m.p. $194.7 \sim 196.2{ }^{\circ} \mathrm{C} ;{ }^{1} \mathrm{H}$ NMR (DMSO- $\left.d_{6}, 300 \mathrm{MHz}\right) \delta: 10.48\left(\mathrm{~s}, 1 \mathrm{H}, \mathrm{SO}_{2} \mathrm{NH}\right), 7.80(\mathrm{~d}$, $J=8.7 \mathrm{~Hz}, 2 \mathrm{H}, \mathrm{ArH}), 7.46$ (d, $J=9.0 \mathrm{~Hz}, 2 \mathrm{H}, \mathrm{ArH}), 7.18$ (d, $J=8.7 \mathrm{~Hz}, 2 \mathrm{H}, \mathrm{ArH}), 6.55(\mathrm{~d}, J=8.4 \mathrm{~Hz}, 2 \mathrm{H}, \mathrm{ArH})$, 6.07 (s, $\left.2 \mathrm{H}, \mathrm{NH}_{2}\right), 4.20\left(\mathrm{t}, J=6.6 \mathrm{~Hz}, 2 \mathrm{H}, \mathrm{CH}_{2}\right), 1.69 \sim$ $1.59\left(\mathrm{~m}, 2 \mathrm{H}, \mathrm{CH}_{2}\right), 1.44 \sim 1.32\left(\mathrm{~m}, 2 \mathrm{H}, \mathrm{CH}_{2}\right), 0.91(\mathrm{t}, J=$ $\left.7.4 \mathrm{~Hz}, 3 \mathrm{H}, \mathrm{CH}_{3}\right)$.

4-[4-(2-氯乙酰氨基) 苯磺酰氨基] 苯甲酸甲酯 (TM2a): 白色固体, 收率 98.1\%. m.p. $242.3 \sim 245.8{ }^{\circ} \mathrm{C}$; ${ }^{1} \mathrm{H}$ NMR (DMSO- $\left.d_{6}, 300 \mathrm{MHz}\right) \delta: 10.82\left(\mathrm{~s}, 1 \mathrm{H}, \mathrm{SO}_{2} \mathrm{NH}\right.$ ), $10.72(\mathrm{~s}, 1 \mathrm{H}, \mathrm{CONH}), 7.84 \sim 7.73(\mathrm{~m}, 6 \mathrm{H}, \operatorname{ArH}), 7.21$ (d, $J=8.1 \mathrm{~Hz}, 2 \mathrm{H}, \mathrm{ArH}), 4.28\left(\mathrm{~s}, 2 \mathrm{H}, \mathrm{CH}_{2}\right), 3.78\left(\mathrm{~s}, 3 \mathrm{H}, \mathrm{CH}_{3}\right)$; ${ }^{13} \mathrm{C}$ NMR (DMSO- $\left.d_{6}, 75 \mathrm{MHz}\right) \delta$ : $165.67,165.39,142.67$, $142.43,133.48,130.64(2 \times \mathrm{C}), 128.18(2 \times \mathrm{C}), 124.33$, $119.20(2 \times \mathrm{C}), 118.16(2 \times \mathrm{C}), 51.95,43.55$; HRMS calcd for $\mathrm{C}_{16} \mathrm{H}_{14} \mathrm{ClN}_{2} \mathrm{O}_{5} \mathrm{~S}[\mathrm{M}-\mathrm{H}]^{-}$381.0317, found 381.0316.

4-[4-(3-氯丙酰氨基) 苯磺酰氨基] 苯甲酸甲酯 (TM2b): 白色固体, 收率 90.1\%. m.p. 228.1 230.8 ${ }^{\circ} \mathrm{C}$; ${ }^{1} \mathrm{H}$ NMR (DMSO- $\left.d_{6}, 300 \mathrm{MHz}\right) \delta: 10.77$ (s, $1 \mathrm{H}, \mathrm{SO}_{2} \mathrm{NH}$ ), $10.46(\mathrm{~s}, 1 \mathrm{H}, \mathrm{CONH}), 7.83 \sim 7.73(\mathrm{~m}, 6 \mathrm{H}, \operatorname{ArH}), 7.21$ (d, $J=8.1 \mathrm{~Hz}, 2 \mathrm{H}, \mathrm{ArH}), 3.85\left(\mathrm{t}, J=6.0 \mathrm{~Hz}, 2 \mathrm{H}, \mathrm{CH}_{2}\right), 3.78$ (s, 3H, $\mathrm{CH}_{3}$ ), 2.84 (t, $\left.J=6.0 \mathrm{~Hz}, 2 \mathrm{H}, \mathrm{CH}_{2}\right) ;{ }^{13} \mathrm{C} \mathrm{NMR}$ (DMSO- $\left.d_{6}, 75 \mathrm{MHz}\right) \delta$ : 168.78, 165.68, 143.09, 142.48, $133.04,130.60(2 \times \mathrm{C}), 128.11(2 \times \mathrm{C}), 124.34,118.91$ $(2 \times \mathrm{C}), 118.18(2 \times \mathrm{C}), 51.91,40.51,39.36$; HRMS calcd for $\mathrm{C}_{17} \mathrm{H}_{17} \mathrm{ClN}_{2} \mathrm{NaO}_{5} \mathrm{~S} \quad[\mathrm{M}+\mathrm{Na}]^{+} 419.0439$, found 419.0452 .

4-[4-(4-氯丁酰氨基) 苯磺酰氨基] 苯甲酸甲酯 (TM2c)：白色固体，收率 $88.8 \%$. m.p. $180.0 \sim 183.4{ }^{\circ} \mathrm{C}$; ${ }^{1} \mathrm{H}$ NMR $\left[\mathrm{CO}\left(\mathrm{CD}_{3}\right)_{2}, 300 \mathrm{MHz}\right] \delta: 7.88(\mathrm{~d}, J=8.1 \mathrm{~Hz}, 2 \mathrm{H}$, ArH), 7.81 (s, 4H, ArH), 7.34 (d, $J=7.5 \mathrm{~Hz}, 2 \mathrm{H}, \operatorname{ArH})$, $3.83\left(\mathrm{~s}, 3 \mathrm{H}, \mathrm{CH}_{3}\right), 3.68\left(\mathrm{t}, J=6.6 \mathrm{~Hz}, 2 \mathrm{H}, \mathrm{CH}_{2}\right), 2.58(\mathrm{t}$, $\left.J=6.9 \mathrm{~Hz}, 2 \mathrm{H}, \mathrm{CH}_{2}\right), 2.15 \sim 2.06\left(\mathrm{~m}, 2 \mathrm{H}, \mathrm{CH}_{2}\right) ;{ }^{13} \mathrm{C} \mathrm{NMR}$ (DMSO- $\left.d_{6}, 75 \mathrm{MHz}\right) \delta$ : 170.43, 165.43, 143.14, 141.85, $132.88,130.21(2 \times \mathrm{C}), 127.89(2 \times \mathrm{C}), 124.80,118.26$ $(2 \times \mathrm{C}), 118.15(2 \times \mathrm{C}), 50.78,43.94,33.08,27.47$; HRMS calcd for $\mathrm{C}_{18} \mathrm{H}_{19} \mathrm{ClN}_{2}-\mathrm{NaO}_{5} \mathrm{~S}[\mathrm{M}+\mathrm{Na}]^{+} 433.0595$, found 433.0598 .

4-(4-丁酰氨基苯磺酰氨基)苯甲酸甲酯(TM2d): 白 色固体, 收率 76.2\%. m.p. 221.8 225.1 ${ }^{\circ} \mathrm{C} ;{ }^{1} \mathrm{H}$ NMR (DMSO- $\left.d_{6}, 300 \mathrm{MHz}\right) \delta: 10.75$ (s, $\left.1 \mathrm{H}, \mathrm{SO}_{2} \mathrm{NH}\right), 10.25$ (s, 1H, CONH), 7.82 (d, $J=9.0 \mathrm{~Hz}, 2 \mathrm{H}, \mathrm{ArH}), 7.75(\mathrm{~s}, 4 \mathrm{H}$, $\operatorname{ArH}), 7.21(\mathrm{~d}, J=8.4 \mathrm{~Hz}, 2 \mathrm{H}, \mathrm{ArH}), 3.78\left(\mathrm{~s}, 3 \mathrm{H}, \mathrm{CH}_{3}\right)$, 2.29 (t, $\left.J=7.5 \mathrm{~Hz}, 2 \mathrm{H}, \mathrm{CH}_{2}\right), 1.65 \sim 1.53\left(\mathrm{~m}, 2 \mathrm{H}, \mathrm{CH}_{2}\right.$ ), 0.89 (t, $J=7.5 \mathrm{~Hz}, 3 \mathrm{H}, \mathrm{CH}_{3}$ ); ${ }^{13} \mathrm{C}$ NMR (DMSO- $d_{6}, 75$ MHz) $\delta$ : 171.94, 165.67, 143.48, 142.51, 132.56, 130.61 $(2 \times \mathrm{C}), 128.05(2 \times \mathrm{C}), 124.24,118.74(2 \times \mathrm{C}), 118.06$ $(2 \times \mathrm{C}), 51.91,38.39,18.40,13.56$; HRMS calcd for $\mathrm{C}_{18} \mathrm{H}_{20} \mathrm{~N}_{2} \mathrm{NaO}_{5} \mathrm{~S}[\mathrm{M}+\mathrm{Na}]^{+}$399.0985, found 399.0988.

4-[4-(2-氯乙酰氨基)苯磺酰氨基] 苯甲酸乙酯 (TM2e): 白色固体，收率 97.2\%. m.p. $214.3 \sim 217.9{ }^{\circ} \mathrm{C}$; ${ }^{1} \mathrm{H}$ NMR (DMSO- $\left.d_{6}, 300 \mathrm{MHz}\right) \delta: 10.80\left(\mathrm{~s}, 1 \mathrm{H}, \mathrm{SO}_{2} \mathrm{NH}\right.$ ), $10.72(\mathrm{~s}, 1 \mathrm{H}, \mathrm{CONH}), 7.83 \sim 7.73(\mathrm{~m}, 6 \mathrm{H}, \operatorname{ArH}), 7.21$ (d, $J=8.1 \mathrm{~Hz}, 2 \mathrm{H}, \mathrm{ArH}), 4.28 \sim 4.22\left(\mathrm{~m}, 4 \mathrm{H}, 2 \times \mathrm{CH}_{2}\right), 1.26$ (t, $\left.J=6.9 \mathrm{~Hz}, 3 \mathrm{H}, \mathrm{CH}_{3}\right) ;{ }^{13} \mathrm{C}$ NMR (DMSO- $\left.d_{6}, 75 \mathrm{MHz}\right) \delta$ : $165.39,165.16,142.65,142.36,133.48,130.57(2 \times \mathrm{C})$, $128.18(2 \times \mathrm{C}), 124.62,119.19(2 \times \mathrm{C}), 118.18(2 \times \mathrm{C})$, $60.50,43.54,14.18$; HRMS calcd for $\mathrm{C}_{17} \mathrm{H}_{16} \mathrm{ClN}_{2} \mathrm{O}_{5} \mathrm{~S}$ $[\mathrm{M}-\mathrm{H}]^{-}$395.0474, found 395.046.

4-[4-(3-氯丙酰氨基) 苯磺酰氨基] 苯甲酸乙酯 (TM2f)：白色固体，收率 89.0\%. m.p. 191.1 193.3 ${ }^{\circ} \mathrm{C}$; ${ }^{1} \mathrm{H}$ NMR (DMSO- $\left.d_{6}, 300 \mathrm{MHz}\right) \delta: 10.75\left(\mathrm{~s}, 1 \mathrm{H}, \mathrm{SO}_{2} \mathrm{NH}\right)$, $10.46(\mathrm{~s}, 1 \mathrm{H}, \mathrm{CONH}), 7.82(\mathrm{~d}, J=8.4 \mathrm{~Hz}, 2 \mathrm{H}, \operatorname{ArH})$, $7.76 \sim 7.73(\mathrm{~m}, 4 \mathrm{H}, \mathrm{ArH}), 7.21(\mathrm{~d}, J=8.4 \mathrm{~Hz}, 2 \mathrm{H}, \operatorname{ArH})$, 4.24 (q, $\left.J=7.2 \mathrm{~Hz}, 2 \mathrm{H}, \mathrm{CH}_{2}\right), 3.86(\mathrm{t}, J=6.0 \mathrm{~Hz}, 2 \mathrm{H}$, $\left.\mathrm{CH}_{2}\right), 2.84\left(\mathrm{t}, J=6.0 \mathrm{~Hz}, 2 \mathrm{H}, \mathrm{CH}_{2}\right), 1.27(\mathrm{t}, J=7.2 \mathrm{~Hz}, 3 \mathrm{H}$, 
$\mathrm{CH}_{3}$ ); ${ }^{13} \mathrm{C}$ NMR (DMSO- $\left.d_{6}, 75 \mathrm{MHz}\right) \delta: 168.80,165.18$, $143.07,142.41,132.89,130.56(2 \times \mathrm{C}), 128.14(2 \times \mathrm{C})$, $124.62,118.88(2 \times \mathrm{C}), 118.16(2 \times \mathrm{C}), 60.50,40.51$, $39.34,14.17$; HRMS calcd for $\mathrm{C}_{18} \mathrm{H}_{19} \mathrm{ClN}_{2} \mathrm{NaO}_{5} \mathrm{~S}[\mathrm{M}+$ $\mathrm{Na}]^{+}$433.0595, found 433.0603 .

4-[4-(4-氯丁酰氨基) 苯磺酰氨基] 苯甲酸乙酯 (TM2g): 白色固体, 收率 74.7\%. m.p. 183.9 187.4 ${ }^{\circ} \mathrm{C}$; ${ }^{1} \mathrm{H}$ NMR (DMSO- $\left.d_{6}, 300 \mathrm{MHz}\right) \delta: 10.74\left(\mathrm{~s}, 1 \mathrm{H}, \mathrm{SO}_{2} \mathrm{NH}\right.$ ), $10.36(\mathrm{~s}, 1 \mathrm{H}, \mathrm{CONH}), 7.81(\mathrm{~d}, J=8.7 \mathrm{~Hz}, 2 \mathrm{H}, \mathrm{ArH})$, $7.77 \sim 7.68(\mathrm{~m}, 4 \mathrm{H}, \mathrm{ArH}), 7.21(\mathrm{~d}, J=9.0 \mathrm{~Hz}, 2 \mathrm{H}, \mathrm{ArH})$, 4.24 (q, $J=7.2 \mathrm{~Hz}, 2 \mathrm{H}, \mathrm{CH}_{2}$ ), 3.68 (t, $J=6.3 \mathrm{~Hz}, 2 \mathrm{H}$, $\mathrm{CH}_{2}$ ), $2.48\left(\mathrm{~s}, 2 \mathrm{H}, \mathrm{CH}_{2}\right), 2.06 \sim 1.99\left(\mathrm{~m}, 2 \mathrm{H}, \mathrm{CH}_{2}\right), 1.27$ (t, $\left.J=6.9 \mathrm{~Hz}, 3 \mathrm{H}, \mathrm{CH}_{3}\right) ;{ }^{13} \mathrm{C}$ NMR (DMSO- $\left.d_{6}, 75 \mathrm{MHz}\right) \delta$ : $171.04,165.18,143.35,142.48,132.66,130.52(2 \times \mathrm{C})$, $128.05(2 \times \mathrm{C}), 124.55,118.78(2 \times \mathrm{C}), 118.15(2 \times \mathrm{C})$, $60.49,44.90,33.49,27.71,14.09$; HRMS calcd for $\mathrm{C}_{19} \mathrm{H}_{21} \mathrm{ClN}_{2} \mathrm{NaO}_{5} \mathrm{~S}[\mathrm{M}+\mathrm{Na}]^{+}$447.0752, found 447.0754.

4-(4-丁酰氨基苯磺酰氨基)苯甲酸乙酯(TM2h): 白 色固体, 收率 70.4\%. m.p. 205.0 207.5 ${ }^{\circ} \mathrm{C} ;{ }^{1} \mathrm{H}$ NMR (DMSO- $\left.d_{6}, 300 \mathrm{MHz}\right) \delta: 10.73$ (s, $1 \mathrm{H}, \mathrm{SO}_{2} \mathrm{NH}$ ), 10.25 (s, 1H, CONH), 7.81 (d, J=8.7 Hz, 2H, ArH), 7.74 (s, 4H, ArH), 7.20 (d, $J=8.4 \mathrm{~Hz}, 2 \mathrm{H}, \operatorname{ArH}), 4.24$ (q, $J=6.9 \mathrm{~Hz}$, $\left.2 \mathrm{H}, \mathrm{CH}_{2}\right), 2.29$ (t, $\left.J=6.9 \mathrm{~Hz}, 2 \mathrm{H}, \mathrm{CH}_{2}\right), 1.65 \sim 1.53(\mathrm{~m}$, $\left.2 \mathrm{H}, \mathrm{CH}_{2}\right), 1.27$ (t, $\left.J=7.2 \mathrm{~Hz}, 3 \mathrm{H}, \mathrm{CH}_{3}\right), 0.89$ (t, $J=6.9 \mathrm{~Hz}$, $\left.3 \mathrm{H}, \mathrm{CH}_{3}\right) ;{ }^{13} \mathrm{C}$ NMR (DMSO- $\left.d_{6}, 75 \mathrm{MHz}\right) \delta: 171.91$, $165.19,143.40,142.80,132.74,130.54(2 \times \mathrm{C}), 128.02$ $(2 \times \mathrm{C}), 124.34,118.70(2 \times \mathrm{C}), 118.11(2 \times \mathrm{C}), 60.45$, 38.38, 18.39, 14.17, 13.56; HRMS calcd for $\mathrm{C}_{19} \mathrm{H}_{21} \mathrm{~N}_{2} \mathrm{O}_{5} \mathrm{~S}$ $[\mathrm{M}-\mathrm{H}]^{-}$389.1177, found 389.1174 .

4-[4-(2-氯乙酰氨基) 苯磺酰氨基] 苯甲酸丙酯 (TM2i): 白色固体, 收率 94.6\%. m.p. $192.5 \sim 195.5{ }^{\circ} \mathrm{C}$; ${ }^{1} \mathrm{H}$ NMR (DMSO- $\left.d_{6}, 300 \mathrm{MHz}\right) \delta: 10.82\left(\mathrm{~s}, 1 \mathrm{H}, \mathrm{SO}_{2} \mathrm{NH}\right.$ ), 10.73 (s, 1H, CONH), $7.83 \sim 7.72(\mathrm{~m}, 6 \mathrm{H}, \mathrm{ArH}), 7.20$ (d, $J=8.4 \mathrm{~Hz}, 2 \mathrm{H}, \mathrm{ArH}), 4.28\left(\mathrm{~s}, 2 \mathrm{H}, \mathrm{CH}_{2}\right), 4.15(\mathrm{t}, J=6.3$ $\left.\mathrm{Hz}, 2 \mathrm{H}, \mathrm{CH}_{2}\right), 1.72 \sim 1.60\left(\mathrm{~m}, 2 \mathrm{H}, \mathrm{CH}_{2}\right), 0.92(\mathrm{t}, J=7.2$ $\left.\mathrm{Hz}, 3 \mathrm{H}, \mathrm{CH}_{3}\right) ;{ }^{13} \mathrm{C}$ NMR (DMSO- $\left.d_{6}, 75 \mathrm{MHz}\right) \delta: 165.38$, $165.21,142.67,142.38,133.50,130.57(2 \times \mathrm{C}), 128.16$ $(2 \times \mathrm{C}), 124.59,119.19(2 \times \mathrm{C}), 118.19(2 \times \mathrm{C}), 65.90$, 43.53, 21.63, 10.34; HRMS calcd for $\mathrm{C}_{18} \mathrm{H}_{18} \mathrm{ClN}_{2} \mathrm{O}_{5} \mathrm{~S}$ $[\mathrm{M}-\mathrm{H}]^{-}$409.0630, found 409.0625.

4-[4-(3-氯丙酰氨基) 苯磺酰氨基] 苯甲酸丙酯 (TM2j): 白色固体, 收率 $82.5 \%$. m.p. $170.8 \sim 176.7{ }^{\circ} \mathrm{C}$; ${ }^{1} \mathrm{H}$ NMR (DMSO- $\left.d_{6}, 300 \mathrm{MHz}\right) \delta: 10.76\left(\mathrm{~s}, 1 \mathrm{H}, \mathrm{SO}_{2} \mathrm{NH}\right.$ ), $10.45(\mathrm{~s}, 1 \mathrm{H}, \mathrm{CONH}), 7.83$ (d, $J=8.7 \mathrm{~Hz}, 2 \mathrm{H}, \mathrm{ArH})$, $7.76 \sim 7.73$ (m, 4H, ArH), 7.21 (d, $J=8.1 \mathrm{~Hz}, 2 \mathrm{H}, \mathrm{ArH})$,
4.15 (t, $\left.J=6.3 \mathrm{~Hz}, 2 \mathrm{H}, \mathrm{CH}_{2}\right), 3.86\left(\mathrm{t}, J=6.3 \mathrm{~Hz}, 2 \mathrm{H}, \mathrm{CH}_{2}\right)$, $2.84\left(\mathrm{t}, J=6.0 \mathrm{~Hz}, 2 \mathrm{H}, \mathrm{CH}_{2}\right), 1.72 \sim 1.61\left(\mathrm{~m}, 2 \mathrm{H}, \mathrm{CH}_{2}\right)$, $0.93\left(\mathrm{t}, J=7.2 \mathrm{~Hz}, 3 \mathrm{H}, \mathrm{CH}_{3}\right.$ ); ${ }^{13} \mathrm{C}$ NMR (DMSO- $d_{6}, 75$ MHz) $\delta: 168.78,165.22,143.07,142.44,133.06,130.55$ $(2 \times \mathrm{C}), 128.11(2 \times \mathrm{C}), 124.62,118.90(2 \times \mathrm{C}), 118.19$ $(2 \times \mathrm{C}), 65.90,40.50,39.36,21.63,10.34$; HRMS calcd for $\mathrm{C}_{19} \mathrm{H}_{21} \mathrm{ClN}_{2} \mathrm{NaO}_{5} \mathrm{~S}[\mathrm{M}+\mathrm{Na}]^{+}$447.0752, found 447.0751.

4-[4-(4-氯丁酰氨基) 苯磺酰氨基] 苯甲酸丙酯 (TM2k): 白色固体, 收率 88.0\%. m.p. $169.0 \sim 173.8{ }^{\circ} \mathrm{C}$; ${ }^{1} \mathrm{H}$ NMR (DMSO- $\left.d_{6}, 300 \mathrm{MHz}\right) \delta$ : $10.74\left(\mathrm{~s}, 1 \mathrm{H}, \mathrm{SO}_{2} \mathrm{NH}\right)$, $10.36(\mathrm{~s}, 1 \mathrm{H}, \mathrm{CONH}), 7.82(\mathrm{~d}, J=8.4 \mathrm{~Hz}, 2 \mathrm{H}, \mathrm{ArH})$, $7.74 \sim 7.72(\mathrm{~m}, 4 \mathrm{H}, \mathrm{ArH}), 7.21(\mathrm{~d}, J=7.8 \mathrm{~Hz}, 2 \mathrm{H}, \mathrm{ArH})$, $4.16\left(\mathrm{t}, J=6.9 \mathrm{~Hz}, 2 \mathrm{H}, \mathrm{CH}_{2}\right), 3.68\left(\mathrm{t}, J=6.6 \mathrm{~Hz}, 2 \mathrm{H}, \mathrm{CH}_{2}\right)$, $2.51 \sim 2.47\left(\mathrm{~m}, 2 \mathrm{H}, \mathrm{CH}_{2}\right), 2.06 \sim 1.97\left(\mathrm{~m}, 2 \mathrm{H}, \mathrm{CH}_{2}\right)$, $1.73 \sim 1.61\left(\mathrm{~m}, 2 \mathrm{H}, \mathrm{CH}_{2}\right), 0.93\left(\mathrm{t}, J=7.2 \mathrm{~Hz}, 3 \mathrm{H}, \mathrm{CH}_{3}\right)$; ${ }^{13} \mathrm{C}$ NMR (DMSO- $\left.d_{6}, 75 \mathrm{MHz}\right) \delta: 170.98,165.23,143.36$, $142.48,132.75,130.54(2 \times \mathrm{C}), 128.05(2 \times \mathrm{C}), 124.58$, $118.81(2 \times \mathrm{C}), 118.16(2 \times \mathrm{C}), 65.90,44.90,33.52,27.70$, 21.65, 10.33; HRMS calcd for $\mathrm{C}_{20} \mathrm{H}_{23} \mathrm{ClN}_{2} \mathrm{NaO}_{5} \mathrm{~S}[\mathrm{M}+$ $\mathrm{Na}]^{+}$461.0908, found 461.0917.

4-(4-丁酰氨基苯磺酰氨基)苯甲酸丙酯(TM2I): 白 色固体, 收率 71.9\%. m.p. 182.7 184.4 ${ }^{\circ} \mathrm{C} ;{ }^{1} \mathrm{H}$ NMR (DMSO- $\left.d_{6}, 300 \mathrm{MHz}\right) \delta: 10.74\left(\mathrm{~s}, 1 \mathrm{H}, \mathrm{SO}_{2} \mathrm{NH}\right), 10.26(\mathrm{~s}$, $1 \mathrm{H}, \mathrm{CONH}), 7.82(\mathrm{~d}, J=8.4 \mathrm{~Hz}, 2 \mathrm{H}, \mathrm{ArH}), 7.74(\mathrm{~s}, 4 \mathrm{H}$, ArH), 7.20 (d, $J=9.0 \mathrm{~Hz}, 2 \mathrm{H}, \operatorname{ArH}), 4.15$ (t, $J=6.6 \mathrm{~Hz}$, $\left.2 \mathrm{H}, \mathrm{CH}_{2}\right), 2.29\left(\mathrm{t}, J=7.2 \mathrm{~Hz}, 2 \mathrm{H}, \mathrm{CH}_{2}\right), 1.70 \sim 1.55(\mathrm{~m}$, $\left.4 \mathrm{H}, 2 \mathrm{CH}_{2}\right), \quad 0.95 \sim 0.86\left(\mathrm{~m}, 6 \mathrm{H}, 2 \mathrm{CH}_{3}\right) ;{ }^{13} \mathrm{C} \quad \mathrm{NMR}$ $\left(\right.$ DMSO- $\left._{6}, 75 \mathrm{MHz}\right) \delta: 171.91,165.23,143.35,142.72$, $130.54,129.00(2 \times \mathrm{C}), 128.04(2 \times \mathrm{C}), 124.37,118.72$ $(2 \times \mathrm{C}), 118.11(2 \times \mathrm{C}), 65.89,38.39,21.64,18.39,13.56$, 10.34; HRMS calcd for $\mathrm{C}_{20} \mathrm{H}_{24} \mathrm{~N}_{2} \mathrm{NaO}_{5} \mathrm{~S}[\mathrm{M}+\mathrm{Na}]^{+}$ 427.1298, found 427.1302.

4-[4-(2-氯乙酰氨基) 苯磺酰氨基] 苯甲酸丁酯 (TM2m): 白色固体, 收率 93.1\%. m.p. 176.5 178.7 ${ }^{\circ} \mathrm{C}$; ${ }^{1} \mathrm{H}$ NMR (DMSO- $\left.d_{6}, 300 \mathrm{MHz}\right) \delta: 10.81\left(\mathrm{~s}, 1 \mathrm{H}, \mathrm{SO}_{2} \mathrm{NH}\right.$ ), 10.70 (s, 1H, CONH), 7.83 7.73 (m, 6H, ArH), 7.21 (d, $J=8.7 \mathrm{~Hz}, 2 \mathrm{H}, \mathrm{ArH}), 4.28\left(\mathrm{~s}, 2 \mathrm{H}, \mathrm{CH}_{2}\right), 4.20$ (t, $J=6.6$ $\left.\mathrm{Hz}, 2 \mathrm{H}, \mathrm{CH}_{2}\right), 1.68 \sim 1.59\left(\mathrm{~m}, 2 \mathrm{H}, \mathrm{CH}_{2}\right), 1.44 \sim 1.32(\mathrm{~m}$, $\left.2 \mathrm{H}, \mathrm{CH}_{2}\right), 0.90\left(\mathrm{t}, J=7.5 \mathrm{~Hz}, 3 \mathrm{H}, \mathrm{CH}_{3}\right) ;{ }^{13} \mathrm{C} \mathrm{NMR}$ (DMSO- $\left.d_{6}, 75 \mathrm{MHz}\right) \delta$ : $165.37,165.19,142.64,142.42$, $133.55,130.57(2 \times \mathrm{C}), 128.18(2 \times \mathrm{C}), 124.57,119.20$ $(2 \times \mathrm{C}), 118.17(2 \times \mathrm{C}), 64.16,43.55,30.26,18.75,13.60$; HRMS calcd for $\mathrm{C}_{19} \mathrm{H}_{20} \mathrm{ClN}_{2} \mathrm{O}_{5} \mathrm{~S}[\mathrm{M}-\mathrm{H}]^{-}$423.0787, found 423.0782 .

4-[4-(3-氯丙酰氨基) 苯磺酰氨基] 苯甲酸丁酯 
(TM2n): 白色固体，收率 76.7\%. m.p. 168.3 172.4 ${ }^{\circ} \mathrm{C}$; ${ }^{1} \mathrm{H}$ NMR (DMSO- $\left.d_{6}, 300 \mathrm{MHz}\right) \delta: 10.75\left(\mathrm{~s}, 1 \mathrm{H}, \mathrm{SO}_{2} \mathrm{NH}\right.$ ), $10.45(\mathrm{~s}, 1 \mathrm{H}, \mathrm{CONH}), 7.81(\mathrm{~d}, J=8.1 \mathrm{~Hz}, 2 \mathrm{H}, \operatorname{ArH})$, $7.77 \sim 7.73(\mathrm{~m}, 4 \mathrm{H}, \mathrm{ArH}), 7.20(\mathrm{~d}, J=8.7 \mathrm{~Hz}, 2 \mathrm{H}, \mathrm{ArH})$, $4.20\left(\mathrm{t}, J=6.9 \mathrm{~Hz}, 2 \mathrm{H}, \mathrm{CH}_{2}\right), 3.86\left(\mathrm{t}, J=6.3 \mathrm{~Hz}, 2 \mathrm{H}, \mathrm{CH}_{2}\right)$, $2.84\left(\mathrm{t}, J=6.3 \mathrm{~Hz}, 2 \mathrm{H}, \mathrm{CH}_{2}\right), 1.68 \sim 1.59\left(\mathrm{~m}, 2 \mathrm{H}, \mathrm{CH}_{2}\right)$, $1.44 \sim 1.32\left(\mathrm{~m}, 2 \mathrm{H}, \mathrm{CH}_{2}\right), 0.90\left(\mathrm{t}, J=7.2 \mathrm{~Hz}, 3 \mathrm{H}, \mathrm{CH}_{3}\right)$; ${ }^{13} \mathrm{C}$ NMR (DMSO- $\left.d_{6}, 75 \mathrm{MHz}\right) \delta: 168.80,165.21,143.14$, $142.28,133.00,130.56(2 \times \mathrm{C}), 128.12(2 \times \mathrm{C}), 124.57$, $118.88(2 \times \mathrm{C}), 118.15(2 \times \mathrm{C}), 64.16,40.52,39.51,30.26$, 18.73, 13.60; HRMS calcd for $\mathrm{C}_{20} \mathrm{H}_{23} \mathrm{ClN}_{2} \mathrm{NaO}_{5} \mathrm{~S}$ 461.0908, found $[\mathrm{M}+\mathrm{Na}]^{+} 461.0908$.

4-[4-(4-氯丁酰氨基) 苯磺酰氨基] 苯甲酸丁酯 (TM2o): 白色固体, 收率 84.6\%. m.p. 153.0 156.0 ${ }^{\circ} \mathrm{C}$; ${ }^{1} \mathrm{H}$ NMR (DMSO- $\left.d_{6}, 300 \mathrm{MHz}\right) \delta: 10.74\left(\mathrm{~s}, 1 \mathrm{H}, \mathrm{SO}_{2} \mathrm{NH}\right.$ ), $10.36(\mathrm{~s}, 1 \mathrm{H}, \mathrm{CONH}), 7.82(\mathrm{~d}, J=8.4 \mathrm{~Hz}, 2 \mathrm{H}, \mathrm{ArH})$, $7.75 \sim 7.72(\mathrm{~m}, 4 \mathrm{H}, \mathrm{ArH}), 7.21(\mathrm{~d}, J=7.8 \mathrm{~Hz}, 2 \mathrm{H}, \mathrm{ArH})$, $4.20\left(\mathrm{t}, J=6.3 \mathrm{~Hz}, 2 \mathrm{H}, \mathrm{CH}_{2}\right), 3.68\left(\mathrm{t}, J=6.3 \mathrm{~Hz}, 2 \mathrm{H}, \mathrm{CH}_{2}\right)$, $2.51 \sim 2.46\left(\mathrm{~m}, 2 \mathrm{H}, \mathrm{CH}_{2}\right), 2.06 \sim 1.97\left(\mathrm{~m}, 2 \mathrm{H}, \mathrm{CH}_{2}\right)$, $1.68 \sim 1.59\left(\mathrm{~m}, 2 \mathrm{H}, \mathrm{CH}_{2}\right), 1.44 \sim 1.32\left(\mathrm{~m}, 2 \mathrm{H}, \mathrm{CH}_{2}\right), 0.90$ (t, $J=7.2 \mathrm{~Hz}, 3 \mathrm{H}, \mathrm{CH}_{3}$ ); ${ }^{13} \mathrm{C}$ NMR (DMSO- $\left.d_{6}, 75 \mathrm{MHz}\right) \delta$ : $171.31,165.21,143.59,142.50,132.66,130.53(2 \times \mathrm{C})$, $128.05(2 \times \mathrm{C}), 124.60,118.78(2 \times \mathrm{C}), 118.13(2 \times \mathrm{C})$, $64.10,44.90,33.49,30.26,27.71,18.74,13.59$; HRMS calcd for $\mathrm{C}_{21} \mathrm{H}_{25} \mathrm{ClN}_{2} \mathrm{NaO}_{5} \mathrm{~S}[\mathrm{M}+\mathrm{Na}]^{+} 475.1065$, found 475.1072.

4-(4-丁酰氨基苯磺酰氨基)苯甲酸丁酯(TM2p): 白 色固体, 收率 $64.1 \%$. m.p. $181.5 \sim 183.5{ }^{\circ} \mathrm{C} ;{ }^{1} \mathrm{H}$ NMR (DMSO- $\left.d_{6}, 300 \mathrm{MHz}\right) \delta: 10.74\left(\mathrm{~s}, 1 \mathrm{H}, \mathrm{SO}_{2} \mathrm{NH}\right), 10.25(\mathrm{~s}$, $1 \mathrm{H}, \mathrm{CONH}), 7.82(\mathrm{~d}, J=8.4 \mathrm{~Hz}, 2 \mathrm{H}, \mathrm{ArH}), 7.76 \sim 7.70$ (m, 4H, ArH), 7.21 (d, $J=8.1 \mathrm{~Hz}, 2 \mathrm{H}, \operatorname{ArH}), 4.20$ (t, $J=6.6$ $\mathrm{Hz}, 2 \mathrm{H}, \mathrm{CH}_{2}$ ), 2.30 (t, $\left.J=7.2 \mathrm{~Hz}, 2 \mathrm{H}, \mathrm{CH}_{2}\right), 1.66 \sim 1.58$ $\left(\mathrm{m}, 4 \mathrm{H}, 2 \mathrm{CH}_{2}\right), 1.44 \sim 1.32\left(\mathrm{~m}, 2 \mathrm{H}, \mathrm{CH}_{2}\right), 0.93 \sim 0.87(\mathrm{~m}$, $\left.6 \mathrm{H}, 2 \mathrm{CH}_{3}\right) ;{ }^{13} \mathrm{C}$ NMR (DMSO- $\left.d_{6}, 75 \mathrm{MHz}\right) \delta: 171.89$, $165.18,143.49,142.47,132.58,130.55(2 \times \mathrm{C}), 128.04$
$(2 \times \mathrm{C}), 124.49,118.70(2 \times \mathrm{C}), 118.07(2 \times \mathrm{C}), 64.12$, $38.40,30.26,18.72,18.39,13.57(2 \times \mathrm{C})$; HRMS calcd for $\mathrm{C}_{21} \mathrm{H}_{26} \mathrm{~N}_{2} \mathrm{NaO}_{5} \mathrm{~S}[\mathrm{M}+\mathrm{Na}]^{+}$441.1455, found 441.1455.

\section{2 结果与讨论}

\section{1 实验结果}

前述合成实验的结果见表 1 3. PPRE-Luc 报告基 因篮选实验结果见表 4.

\section{2 结果讨论}

\subsection{1 目标分子合成路线设计}

目标分子 TM2 可认为由四部分组成: PABA 及磺胺 片段, 酯烷基 $\mathrm{R}^{2}$ 及修饰基 $\mathrm{R}^{1} \mathrm{CO}$. 合成 $\mathrm{TM2}$ 涉及各片段 的引入顺序问题. 基于前期研究结果和简捷高效的要 求, 先以 ASC 和 PABA 直接偶联构建基本骨架 IM1 最 为合理. IM1 脱乙酰基即得重要中间体 IM2. 其后, 涉 及先酯化还是先酰基化的选择, 亦即本研究的先酯化策 略或先酰基化策略. 最初, 作者设计了路线 A (Scheme 3).

路线 A 经 3 步反应高收率得到目标产物 TM1. 将 TM1 硫酸催化酯化, 总有少量酰基脱除的产物; TM1 以 二氯亚砜和醇酯化, 不能得到 TM2, 反而得到中间体 IM3, 这就是路线 D, 这是路线最长的 TM2 制备路线; 若用卤代烃和 TM1 的亲核取代反应制备 TM2, 则涉及 相对价昂的卤代烃. 因此，作者放弃了以路线 A 制备 TM2.

路线 C 是直接将 IM2 先酯化(获取 IM3) 再酰化来制 备 TM2, 虽然实验顺利收率很好, 但有 4 步反应. 受 TM1 经 $\mathrm{SOCl}_{2} / \mathrm{ROH}$ 酯化时左端酰基易脱落的启示, 作 者设计了 3 步反应的路线 B (Scheme 4).

实验证明，路线 A 和 B 分别是制备 TM1 和 TM2 的最优路线.

本研究进一步证实，二氯亚砜和醇在酯化羧基的同 时能够将脂肪酰芳胺的酰基脱除, 二氯亚砜/醇体系是 羧酸酯和脂肪酰芳胺共存时的选择性脱酰基试剂.

表 1 中间体 IM2 的制备

Table 1 Synthetic results of intermediate IM2

\begin{tabular}{|c|c|c|c|c|c|c|c|}
\hline Entry & IM1 $/(\mathrm{g} / \mathrm{mmol})$ & Acid or base $/ \mathrm{mL}$ & Solvent & Time/h & Temp. $/{ }^{\circ} \mathrm{C}$ & Weight/g & Yield/\% \\
\hline 1 & $1.7 / 5.1$ & Conc. $\mathrm{HCl} / 12.0$ & EtOH & 4 & 78 & 0.2 & 16 \\
\hline 2 & $1.7 / 5.0$ & Conc. $\mathrm{HCl} / 40.0$ & $\mathrm{EtOH}$ & 6 & 78 & 0.1 & 5 \\
\hline 3 & $0.3 / 1.0$ & $2 \mathrm{~mol} \cdot \mathrm{L}^{-1} \mathrm{KOH} / 10.0$ & $\mathrm{H}_{2} \mathrm{O}$ & 9 & 40 & 0.2 & 72 \\
\hline 4 & $0.7 / 2.0$ & Conc. $\mathrm{HCl} / 20.0$ & $\mathrm{Me}_{2} \mathrm{CO}$ & 26 & 59 & 0.4 & 70 \\
\hline 5 & $0.7 / 1.9$ & Conc. $\mathrm{HCl} / 20.0$ & THF & 26 & 68 & 0.4 & 70 \\
\hline 6 & $11.0 / 32.8$ & $2 \mathrm{~mol} \cdot \mathrm{L}^{-1} \mathrm{KOH} / 50.0$ & $\mathrm{H}_{2} \mathrm{O}$ & 23 & 50 & 7.8 & 81 \\
\hline 7 & $8.8 / 26.4$ & $2 \mathrm{~mol} \cdot \mathrm{L}^{-1} \mathrm{KOH} / 50.0$ & $\mathrm{H}_{2} \mathrm{O}$ & 33 & 50 & 7.3 & 95 \\
\hline
\end{tabular}


表 2 中间体 IM3 的制备

Table 2 Synthesis of intermediate IM3

\begin{tabular}{|c|c|c|c|c|c|c|c|c|c|c|}
\hline \multirow{2}{*}{ Entry } & \multirow{2}{*}{ IM } & \multirow{2}{*}{$\mathrm{R}^{2}$} & \multicolumn{2}{|c|}{ IM1, IM2 or TM1 } & \multirow{2}{*}{$\mathrm{SOCl}_{2} /(\mathrm{g} / \mathrm{mmol})$} & \multirow{2}{*}{$\mathrm{R}^{2} \mathrm{OH} / \mathrm{mL}$} & \multirow{2}{*}{ Time/h } & \multirow{2}{*}{ Temp. $/{ }^{\circ} \mathrm{C}$} & \multirow{2}{*}{ Weight/g } & \multirow{2}{*}{ Yield/\% } \\
\hline & & & $\mathrm{R}^{1} \mathrm{CO}$ & $\mathrm{g} / \mathrm{mmol}$ & & & & & & \\
\hline 1 & \multirow{6}{*}{$\mathbf{3 a}$} & \multirow{6}{*}{$\mathrm{Me}$} & $\mathrm{H}$ & $0.6 / 2.1$ & $0.4 / 2.9$ & 8 & 9 & 68 & 0.6 & 96 \\
\hline 2 & & & $\mathrm{MeCO}$ & $3.3 / 10.0$ & $1.5 / 12.8$ & 15 & 17 & 65 & 2.8 & 91 \\
\hline 3 & & & $\mathrm{Me}\left(\mathrm{CH}_{2}\right)_{2} \mathrm{CO}$ & $0.4 / 1.0$ & $0.2 / 1.5$ & 8 & 5 & 65 & 0.2 & 79 \\
\hline 4 & & & $\mathrm{ClCH}_{2} \mathrm{CO}$ & $0.4 / 1.0$ & $0.2 / 1.5$ & 6 & 8 & 65 & 0.3 & 95 \\
\hline 5 & & & $\mathrm{Cl}\left(\mathrm{CH}_{2}\right)_{2} \mathrm{CO}$ & $0.8 / 2.0$ & $0.4 / 3.1$ & 10 & 10 & 65 & 0.6 & 97 \\
\hline 6 & & & $\mathrm{Cl}\left(\mathrm{CH}_{2}\right)_{3} \mathrm{CO}$ & $0.8 / 2.0$ & $0.4 / 3.0$ & 10 & 12 & 65 & 0.5 & 82 \\
\hline 7 & \multirow{5}{*}{$\mathbf{3 b}$} & \multirow{5}{*}{ Et } & $\mathrm{MeCO}$ & $2.0 / 6.0$ & $1.0 / 8.4$ & 10 & 9.5 & 75 & 1.7 & 89 \\
\hline 8 & & & $\mathrm{Me}\left(\mathrm{CH}_{2}\right)_{2} \mathrm{CO}$ & $0.3 / 0.8$ & $0.2 / 1.6$ & 8 & 11 & 75 & 0.2 & 83 \\
\hline 9 & & & $\mathrm{ClCH}_{2} \mathrm{CO}$ & $0.4 / 1.0$ & $0.2 / 1.5$ & 10 & 12 & 80 & 0.3 & 91 \\
\hline 10 & & & $\mathrm{Cl}\left(\mathrm{CH}_{2}\right)_{2} \mathrm{CO}$ & $0.4 / 1.0$ & $0.8 / 6.8$ & 10 & 8 & 80 & 0.3 & 95 \\
\hline 11 & & & $\mathrm{Cl}\left(\mathrm{CH}_{2}\right)_{3} \mathrm{CO}$ & $0.4 / 1.0$ & $0.2 / 1.6$ & 8 & 9 & 80 & 0.3 & 84 \\
\hline 12 & \multirow{5}{*}{$3 \mathrm{c}$} & \multirow{5}{*}{$n-\operatorname{Pr}$} & $\mathrm{MeCO}$ & $3.3 / 10.0$ & $1.5 / 12.8$ & 15 & 11 & 86 & 3.0 & 90 \\
\hline 13 & & & $\mathrm{Me}\left(\mathrm{CH}_{2}\right)_{2} \mathrm{CO}$ & $0.3 / 0.9$ & $0.2 / 1.6$ & 6 & 12 & 81 & 0.3 & 82 \\
\hline 14 & & & $\mathrm{ClCH}_{2} \mathrm{CO}$ & $0.3 / 0.8$ & $0.2 / 1.5$ & 6 & 8 & 86 & 0.3 & 89 \\
\hline 15 & & & $\mathrm{Cl}\left(\mathrm{CH}_{2}\right)_{2} \mathrm{CO}$ & $0.4 / 1.0$ & $0.2 / 1.8$ & 6 & 6 & 86 & 0.3 & 94 \\
\hline 16 & & & $\mathrm{Cl}\left(\mathrm{CH}_{2}\right)_{3} \mathrm{CO}$ & $0.4 / 0.9$ & $0.2 / 1.7$ & 6 & 11 & 84 & 0.2 & 81 \\
\hline 17 & \multirow{6}{*}{ 3d } & \multirow{6}{*}{$n-\mathrm{Bu}$} & $\mathrm{H}$ & $0.6 / 2.0$ & $0.4 / 3.0$ & 10 & 10 & 83 & 0.7 & 93 \\
\hline 18 & & & $\mathrm{MeCO}$ & $3.3 / 10.0$ & $1.6 / 13.5$ & 15 & 11 & 82 & 3.1 & 89 \\
\hline 19 & & & $\mathrm{Me}\left(\mathrm{CH}_{2}\right)_{2} \mathrm{CO}$ & $0.394 / 1.1$ & $0.198 / 1.6$ & 7 & 18 & 86 & 0.3 & 88 \\
\hline 20 & & & $\mathrm{ClCH}_{2} \mathrm{CO}$ & $0.281 / 0.7$ & $0.198 / 1.7$ & 7 & 13 & 86 & 0.2 & 88 \\
\hline 21 & & & $\mathrm{Cl}\left(\mathrm{CH}_{2}\right)_{2} \mathrm{CO}$ & $0.404 / 1.1$ & $0.222 / 1.9$ & 7 & 12 & 86 & 0.3 & 91 \\
\hline 22 & & & $\mathrm{Cl}\left(\mathrm{CH}_{2}\right)_{3} \mathrm{CO}$ & $0.406 / 1.0$ & $0.191 / 1.6$ & 7 & 12 & 86 & 0.3 & 82 \\
\hline
\end{tabular}

表 3 目标分子 TM2 的制备

Table 3 Synthetic results of target compounds TM2

\begin{tabular}{|c|c|c|c|c|c|c|c|c|c|}
\hline $\mathbf{T M}$ & $\mathrm{R}^{2}$ & $\mathrm{R}^{1}$ & IM3 $/(\mathrm{g} / \mathrm{mmol})$ & $\mathrm{R}^{1} \mathrm{COCl} /(\mathrm{g} / \mathrm{mmol})$ & $\mathrm{K}_{2} \mathrm{CO}_{3} /(\mathrm{g} / \mathrm{mmol})$ & Time/h & Temp. $/{ }^{\circ} \mathrm{C}$ & Weight/g & Yield/\% \\
\hline $2 a$ & \multirow{4}{*}{$\mathrm{Me}$} & $\mathrm{ClCH}_{2}$ & $0.6 / 2.0$ & $0.3 / 3.0$ & $0.6 / 4.5$ & 12 & 24 & 0.8 & 98 \\
\hline $2 b$ & & $\mathrm{Cl}\left(\mathrm{CH}_{2}\right)_{2}$ & $0.3 / 1.1$ & $0.2 / 1.3$ & $0.2 / 1.5$ & 6 & 17 & 0.3 & 90 \\
\hline $2 c$ & & $\mathrm{Cl}\left(\mathrm{CH}_{2}\right)_{3}$ & $0.6 / 2.1$ & $0.4 / 2.8$ & $0.4 / 3.0$ & 9 & 18 & 0.8 & 89 \\
\hline $2 d$ & & $\mathrm{Me}\left(\mathrm{CH}_{2}\right)_{2}$ & $0.6 / 2.0$ & $0.3 / 2.8$ & $0.4 / 2.9$ & 8 & 18 & 0.6 & 76 \\
\hline $2 e$ & \multirow{4}{*}{ Et } & $\mathrm{ClCH}_{2}$ & $0.6 / 2.0$ & $0.3 / 3.0$ & $0.6 / 4.5$ & 12 & 24 & 0.8 & 97 \\
\hline $2 f$ & & $\mathrm{Cl}\left(\mathrm{CH}_{2}\right)_{2}$ & $0.3 / 1.1$ & $0.2 / 1.4$ & $0.2 / 1.6$ & 7 & 14 & 0.4 & 89 \\
\hline $2 \mathrm{~g}$ & & $\mathrm{Cl}\left(\mathrm{CH}_{2}\right)_{3}$ & $0.6 / 2.0$ & $0.4 / 2.7$ & $0.4 / 3.0$ & 12.5 & 18 & 0.6 & 75 \\
\hline $2 \mathrm{~h}$ & & $\mathrm{Me}\left(\mathrm{CH}_{2}\right)_{2}$ & $0.5 / 1.5$ & $0.3 / 2.6$ & $0.3 / 2.2$ & 8 & 19 & 0.4 & 70 \\
\hline $2 \mathbf{i}$ & \multirow{4}{*}{$n-\operatorname{Pr}$} & $\mathrm{ClCH}_{2}$ & $0.7 / 2.0$ & $0.3 / 3.0$ & $0.6 / 4.5$ & 14 & 24 & 0.8 & 95 \\
\hline $2 \mathbf{j}$ & & $\mathrm{Cl}\left(\mathrm{CH}_{2}\right)_{2}$ & $0.7 / 2.0$ & $0.3 / 2.6$ & $0.4 / 3.0$ & 6 & 17 & 0.9 & 83 \\
\hline $2 \mathrm{k}$ & & $\mathrm{Cl}\left(\mathrm{CH}_{2}\right)_{3}$ & $0.5 / 1.4$ & $0.3 / 2.2$ & $0.3 / 2.1$ & 16 & 16 & 0.6 & 88 \\
\hline 21 & & $\mathrm{Me}\left(\mathrm{CH}_{2}\right)_{2}$ & $0.6 / 2.0$ & $0.3 / 2.8$ & $0.4 / 2.9$ & 10 & 18 & 0.5 & 72 \\
\hline $2 m$ & \multirow{4}{*}{$n-\mathrm{Bu}$} & $\mathrm{ClCH}_{2}$ & $0.7 / 2.0$ & $0.3 / 3.0$ & $0.6 / 4.5$ & 14 & 24 & 0.8 & 93 \\
\hline $2 n$ & & $\mathrm{Cl}\left(\mathrm{CH}_{2}\right)_{2}$ & $0.6 / 1.6$ & $0.3 / 2.1$ & $0.3 / 2.4$ & 6 & 17 & 0.5 & 77 \\
\hline 20 & & $\mathrm{Cl}\left(\mathrm{CH}_{2}\right)_{3}$ & $0.7 / 2.0$ & $0.4 / 2.6$ & $0.4 / 3.0$ & 7 & 18 & 0.8 & 85 \\
\hline $2 p$ & & $\mathrm{Me}\left(\mathrm{CH}_{2}\right)_{2}$ & $0.5 / 1.5$ & $0.2 / 2.2$ & $0.3 / 2.2$ & 10 & 19 & 0.4 & 64 \\
\hline
\end{tabular}

\subsubsection{PABA 磺酰化}

文献报道的氨/胺基磺酰化方法很多, 诸如不同条 件下的磺酰氯接胺法 ${ }^{[30,31]}$ 、統基氧化成磺酰氯后接胺 法 ${ }^{[32]}$ 、磺酸酯胺交换法 ${ }^{[33]}$ 和亚砜氧化制备磺胺法 ${ }^{[34]}$. PABA 与对乙酰氨基苯磺酰氯的反应, 目前一般采用直
接缩合法 ${ }^{[35 ~ 37]}$, 不同之处在于溶剂和碱等的差别. 作者 以碳酸钾缚酸、丙酮为溶剂, 室温摚拌反应, 可以一步 合成中间体 IM1，具有收率高、纯度好及廉价简便的优 点. 实验显示, 在选定的条件下, 反应 $10 \mathrm{~h}$ 的收率可以 超过 $90 \%$. 
表 4 中间体及目标化合物的 PPAR 活性数据 ${ }^{a}$

Table 4 PPAR activation activity of intermediates and target compounds

\begin{tabular}{|c|c|c|c|c|c|c|c|c|c|c|c|c|c|}
\hline Compd. & IM1 & IM2 & TM1a & TM1b & TM1c & TM1d & IM3a & IM3b & IM3c & IM3d & TM2a & TM2b & TM2c \\
\hline Conc./(nmol• $\left.\mathrm{L}^{-1}\right)$ & 29.9 & 34.2 & 27.1 & 26.1 & 25.2 & 27.6 & 32.6 & 31.2 & 30.0 & 28.7 & 26.2 & 25.2 & 24.3 \\
\hline $\operatorname{PPAR}^{1} / \%$ & 21.09 & 23.61 & 14.43 & 29.23 & 15.84 & 13.40 & -12.87 & 15.84 & 6.37 & -9.39 & -8.84 & -4.14 & 9.92 \\
\hline $\mathrm{PPAR}^{2} / \%$ & 15.59 & 15.26 & 11.77 & 24.75 & 13.89 & 10.73 & -8.72 & 11.22 & 4.69 & -7.23 & -7.46 & -3.63 & 9.02 \\
\hline Compd. & TM2d & TM2e & TM2f & TM2g & TM2h & TM2i & $\mathbf{T M} \mathbf{j} \mathbf{j}$ & TM2k & TM2I & TM2m & TM2n & TM20 & TM2p \\
\hline Conc./(nmol $\left.\cdot \mathrm{L}^{-1}\right)$ & 23.5 & 25.2 & 22.8 & 24.3 & 23.5 & 24.4 & 22.8 & 22.1 & 26.6 & 23.6 & 25.6 & 24.7 & 23.9 \\
\hline $\operatorname{PPAR}^{1} / \%$ & 6.89 & -22.02 & 12.14 & 2.67 & 6.67 & -14.29 & -19.38 & 23.90 & 8.59 & 0.88 & -5.17 & 3.63 & -13.16 \\
\hline $\operatorname{PPAR}^{2} / \%$ & 6.48 & -19.31 & 11.77 & 2.43 & 6.27 & -12.94 & -18.79 & 23.90 & 7.14 & 0.82 & -4.46 & 3. 25 & -12.17 \\
\hline
\end{tabular}

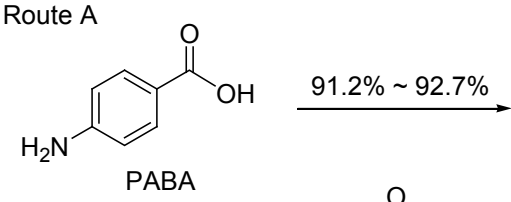<smiles>CC(=O)Nc1ccc(S(=O)(=O)Nc2ccc(C(=O)O)cc2)cc1</smiles>

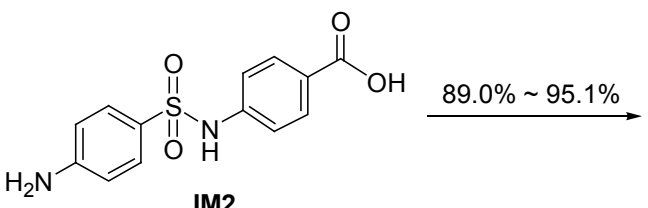<smiles>[R]C(=O)Nc1ccc(S(=O)(=O)Nc2ccc(C(=O)O)cc2)cc1</smiles><smiles>[R]OC(=O)c1ccc(NS(=O)(=O)c2ccc(NC([R])=O)cc2)cc1</smiles>

Scheme 3

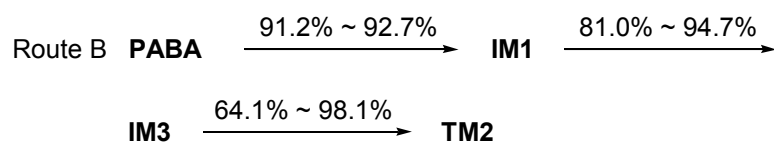

Scheme 4

\subsubsection{IM2 脱酰基}

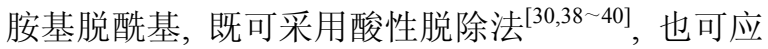
用碱性脱除法 ${ }^{[41,42]}$ 和 DIBAL 脱除法 ${ }^{[43]}$. 作者在 $2 \mathrm{~mol}$ $\mathrm{L}^{-1} \mathrm{KOH}$ 的水溶液中 $50{ }^{\circ} \mathrm{C}$ 加热水解 IM1, 能够方便地 脱除乙酰基; 原料在碱性水溶液中成盐, 溶解性大, 勿 需加入有机溶剂溶解原料; 反应完成后加酸析出, 即可 高收率地得到高纯度 $\mathbf{I M} \mathbf{2}^{[29]}$.

\subsubsection{IM3 酯化}

羧酸酯的制备，文献报道的方法很多，诸如酸催化 的羧酸/醇酯化法 ${ }^{[44]}$ 、羧酸/卤代烃碱促进酯化法 ${ }^{[45,46]}$ 、 酰氯酯化法 ${ }^{[47,48]}$ 、酸酐/醇酸催化酯化法 ${ }^{[49]}$ 、羧酸经 $\mathrm{DCC}^{[50,51]}$ 及 $\mathrm{CDI}^{[51]}$ 促进的酯化法. 二氯亚砜/醇法能将 脱酰基和酯化两步反应一步完成，是本实验室发现的新 方法 ${ }^{[29,30]}$. 本研究继续采用此法, 可以方便地从 IM1 或 TM1 制备 IM3，产物纯收率高.

实验还发现，醇的碳链越长，二氯亚砜/醇法制备对 应酯的收率越低，这可从 IM1 或 IM2 合成 IM3 中得到 证实. TM1 中 $\mathrm{R}^{1}$ 的碳链长度，也影响 TM1 转化为 IM3 的收率，这可能和分子的溶解性、反应物的空间位阻等 因素有关. 虽然如此, 本研究绝大多数酯化反应的收率 超过 $80 \%$ (表 2). 一般而言，反应温度控制在相应醇的沸 点附近或略低于沸点，既有利于反应的快速完成，也保 证反应过程处于液态(反应均匀化).

二氯亚砜/醇法无论是对于氨基裸露的 IM2, 还是 带有乙酰胺基的 IM1，抑或是其它酰基修饰的 TM1，效 果均佳.

\subsubsection{TM1 和 TM2 酰基化}

芳胺酰基化，可采用酰氯法 ${ }^{[52,53]}$ 、酸酐法 ${ }^{[54]}$ 、偶联 法 $^{[26]}$ 和微波辐射辅助合成法 ${ }^{[55]}$. 依照简便易得原则以 及本研究室的经验，采用酰氯法较为合适. 目标分子酰 氯化具有如下特点：(1) TM1 的制备收率很高且易于纯 化; 氯代酰氯比烷基酰氯的收率高(可能和氯原子吸电 子诱导效应有关); 氯代酰氯的碳原子越多, 产物收率 越高(可能和产物溶解性有关). (2) TM2 的合成收率随 $\mathrm{R}^{1}$ 和 $\mathrm{R}^{2}$ 不同而变化. $\mathrm{R}^{2}$ 相同，氯乙酰基修饰所得产物收 率最高(应该是其空间位阻较小所致)，氯代酰氯较烷基 酰氯修饰的产物收率更高(可能仍然与左端氯原子吸电 子能力有关); $\mathrm{R}^{1}$ 相同 $\mathrm{R}^{2}$ 不同时，其收率顺序一般为：甲 酯 $>$ 乙酯 $>$ 丙酯 $>$ 丁酯.

\subsection{PPAR 激动活性}

PPAR 是核受体超家族的一员，与过氧化物酶体增 
殖物的基因转录调节有关 ${ }^{[56]}$, 在调节血糖、胰岛素敏感 性、脂肪咜存与代谢等方面起着至关重要的作用, 被认 为是开发人类代谢疾病治疗药物的重要分子靶标 ${ }^{[3,57]}$. 本研究即是通过测定目标分子的 PPAR 相对活性来考察 目标分子的抗糖尿病活性.

PPAR 相对激动活性结果(表 4 和图 1)显示, 在样品 测试浓度为 $10 \mu \mathrm{g} \cdot \mathrm{mL}^{-1}$ 时, 所合成化合物的 PPAR 活 性都低(不高于 $30 \%$ ); 氨基羧基均未修饰(IM2)时, PPAR 活性值约为 $15 \%$; 氨基游离羧基酯化, 活性均低, 但 IM3b 活性(11.22\%)相对较好; 羧基游离氨基修饰 (TM1 和 IM1)时, 氯丙酰基修饰的 TM1b 活性最好 (24.75\%); 氨基羧基均修饰(TM2)时, 活性差异很大, 丁 酯者活性都差(低于 $12 \%$ ), 但 TM2k (23.90\%), TM2e (19.31\%), TM2j (18.79\%)相对较好; 所有化合物中, TM1b 活性最好. 可能还需要合成更多的分子, 进行更 多的活性测试, 方能发现其结构一活性关系. 进一步的 研究仍在进行中.

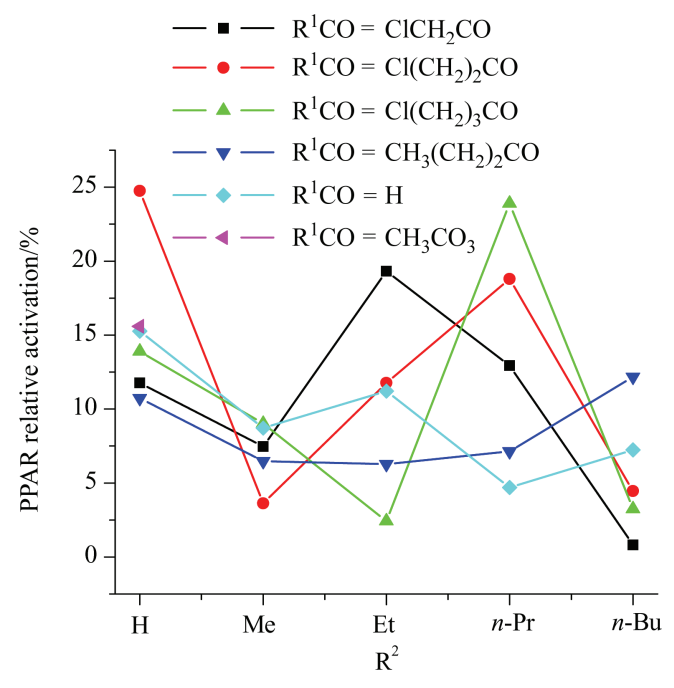

图 1 相同摩尔浓度下的结构-活性关系

Figure 1 PPAR relative activity-substituent diagram

\section{3 结论}

$\mathrm{SOCl}_{2}$ /醇体系对于含有氨基和羧基的分子, 在适宜 温度和投料比下，可以一步脱除脂肪酰芳胺的脂肪酰基 并实现羧基的酯化, 方法简便, 收率很高, 具有较广的 适应性; 借用此方法, 可以通过路线 $\mathrm{B}$ 简便地制备 TM2, 为该类分子的有效合成建立了新的方法. 需要指 出的是, 这些目标分子的生物活性尚有进一步提升的空 间, 值得深入研究.

致谢 西南大学化学化工学院核磁共振室罗群力老师、 龚成斌老师、王宁老师给予帮助, 在此表示衷心的感谢!

\section{References}

[1] Xu, Y. P.; Etgen, G. J.; Broderick, C. L.; Canada, E.; Gonzalez, I.; Lamar, J.; Montrose-Rafizadeh, C.; Oldham, B. A.; Osborne, J. J., Xie, C. Y.; Shi, Q.; Winneroski, L. L.; York, J.; Yumibe, N.; Zink, R.; Mantlo, N. J. Med. Chem. 2006, 49, 5649.

[2] Yoshida, K.; Hishida, A.; Iida, O.; Hosokawa, K.; Kawabata, J. $J$ Agric. Food Chem. 2008, 56, 4367.

[3] Mohler, M. L.; He Y. L.; Wu Z. Z.; Hwang, D. J.; Miller, D. D. Med. Res. Rev. 2009, 29, 125.

[4] Kim, S. J.; Jung, M. H.; Yoo, K. H.; Cho, J. H.; Oh, C. H. Bioorg. Med. Chem. Lett. 2008, 18, 5815.

[5] Temperini, C.; Cecchi, A.; Scozzafava, A.; Supuran, C. T. Bioorg. Med. Chem. Lett. 2008, 18, 2567.

[6] Rothschild, C. M.; Hines, M. T.; Breuhaus, B.; Gay, J.; Sellon, D. C. J. Vet. Intern. Med. 2004, 18, 370 .

[7] Tanaka, H.; Ohshima, N.; Hidaka, H. Mol. Pharmacol. 1999, 55, 356.

[8] Yang, H. T.; Endoh, M. Eur. J. Pharmacol. 1996, 312, 281.

[9] Hao, G. F.; Yang, G. F. Chin. J. Org. Chem. 2008, 28, 1545 (in Chinese).

(郝格非, 杨光富, 有机化学, 2008, 28, 1545.)

[10] Seri, K.; Kazuko, S.; Katsumi, K.; Yorishige, I.; Hiroyuki, A. Eur. J. Pharmacol. 2000, 389, 253.

[11] Basset, G. J. C.; Quinlivan, E. P.; Ravanel, S.; Rébeillé, F.; Nichols, B. P.; Shinozaki, K.; Seki, M.; Adams-Phillips, L. C.; Giovannoni, J. J.; Gregory III, J. F.; Hanson, A. D. Proc. Natl. Acad. Sci. U. S. A. 2004, 101, 1496.

[12] Pawelczak, K.; Jones, T. R.; Kempny, M.; Jackman, A. L.; Newell, D. R.; Krzyzanowski, L.; Rzeszotarska, B. J. Med. Chem. 1989, 32, 160.

[13] Lahue, B. R.; Wan, Z. K.; Snyder, J. K. J. Org. Chem. 2003, 68, 4345.

[14] Ohkanda, J.; Buckner, F. S.; Lockman, J. W.; Yokoyama, K.; Carrico, D.; Eastman, R.; Luca-Fradley, K. D.; Davies, W.; Croft, S. L.; Voorhis, W. C. V.; Gelb, M. H.; Sebti, S. M.; Hamilton, A. D. J. Med. Chem. 2004, 47, 432.

[15] Sun, J.; Yang, Y. S.; Li, W.; Zhang, Y. B.; Wang, X. L.; Tang, J. F.; Zhu, H. L. Bioorg. Med. Chem. Lett. 2011, 21, 6116.

[16] Prabhu, P. P.; Shastry, C. S.; Pande, S.; Pai, A. J. Pharm. Res. 2011, 4, 2209.

[17] Qiu, J. Y.; Xu, B.; Huang, Z. M.; Pan, W. D.; Cao, P. X.; Liu, C. X.; Hao, X. J.; Song, B. A.; Liang, G. Y. Bioorg. Med. Chem. 2011, 19, 5352.

[18] Chen, X. F.; Wu, Y. B.; Jin, J.; Wang, R. Z.; Wang, C.; Liu, J. Acta Pharm. Sin. 2010, 45, 263 (in Chinese).

(陈晓芳, 武燕涁, 金洁, 王瑞贞, 王羽, 刘浚, 药学学报, 2010, 45, 263.)

[19] Mulongo, G.; Mbabazi, J.; Odongkara, B.; Twinomuhwezi, H.; Mpango, G. B. Res. J. Chem. Sci. 2011, 1, 102.

[20] Pfefferkorn, J. A.; Song, Y. T.; Sun, K. L.; Miller, S. R.; Trivedi, B. K.; Choi, C.; Sorenson, R. J.; Bratton, L. D.; Unangst, P. C.; Larsen, S. D.; Poel, T. J.; Cheng, X. M.; Lee, C.; Erasga, N.; Auerbach, B.; Askew, V.; Dillon, L.; Hanselman, J. C.; Lin, Z. W.; Lu, G.; Robertson, A.; Olsen, K.; Mertz, T.; Sekerke, C.; Pavlovsky, A.; Harris, M. S.; Bainbridge, G.; Caspers, N.; Chen, H. F.; Eberstadt, M. Bioorg. Med. Chem. Lett. 2007, 17, 4538.

[21] Dhananjeyan, M. R.; Trendel, J. A.; Bykowski, C.; Sarver, J. G.; Ando, H.; Erhardt, P. W. J. Chromatogr., B 2008, 867, 247.

[22] Yang, D. C.; Yan, J. F.; Xu, J.; Ye, F.; Zhou, Z. W.; Zhang, W. Y.; Fan, L.; Chen, X. Acta Pharm. Sin. 2010, 45, 66 (in Chinese). (杨大成, 晏菊芳, 许莡, 叶飞, 周祖文, 张蔚瑜, 范莉, 陈欣, 
药学学报, 2010, 45, 66.)

[23] Zhang, K.; Yan, J. F.; Tang, X. M.; Liu, H. P.; Fan, L.; Zhou, G. M.; Yang, D. C. Acta Pharm. Sin. 2011, 46, 412 (in Chinese). (张坤, 晏菊芳, 唐雪梅, 刘红萍, 范莉, 周光明, 杨大成, 药学 学报, 2011, 46, 412.)

[24] Tang, X. M.; Yan, J. F.; Zhang, Y. X.; Zhang, W. Y.; Su, X. Y.; Chen, X.; Zhou, Z. W.; Yang, D. C. Chin. J. Org. Chem. 2009, 29, 1790 (in Chinese). (唐雪梅, 晏菊芳, 张映霞, 张蔚瑜, 苏小燕, 陈欣, 周祖文, 杨 大成, 有机化学, 2009, 29, 1790.)

[25] Song, X. L.; Yan, J. F.; Fan, L.; Chen, X.; Xu, J.; Zhou, Z. W.; Yang, D. C. Chin. J. Org. Chem. 2009, 29, 606 (in Chinese). (宋小礼, 晏菊芳, 范莉, 陈欣, 许荩, 周祖文, 杨大成, 有机化 学, 2009, 29, 606.)

[26] Tang, X. M.; Fan, L.; Yu, H. X.; Liao, Y. H.; Yang, D. C. Chin. J. Org. Chem. 2009, 29, 595 (in Chinese). (唐雪梅, 范莉, 于红霞, 廖玉华, 杨大成, 有机化学, 2009, 29, 595.)

[27] Novacek, A.; Sedlackova, V.; Korner, J.; Danek, J. CS 266792, 1990 [Chem. Abstr. 1991, 114, 246957].

[28] Rajagopalan, S. Proc.-Indian Acad. Sci., Sect. A 1943, 18A, 108.

[29] Yang, D. C.; Wang, L. F.; Fan, L.; Wang, G. B.; Shang-Guan, R. Y.; Sun, J.; Li, C. Z.; Yang, L. CN 102153481, 2011 [Chem. Abstr. 2011, 155, 352231].

[30] Wang, G. B.; Wang, L. F.; Li, C. Z.; Sun, J.; Zhou, G. M.; Yang, D. C. Res. Chem. Intermed. 2012, 38, 77.

[31] Meshram, G. A.; Patil, V. D. Tetrahedron Lett. 2009, 50, 1117.

[32] Bahrami, K.; Khodaei, M. M.; Soheilizad, M. J. Org. Chem. 2009, $74,9287$.

[33] Palakurthy, N. B.; Mandal, B. Tetrahedron Lett. 2011, 52, 7132.

[34] Yu, X. F.; Lu, X. Y. Adv. Synth. Catal. 2011, 353, 2805.

[35] Luo, X. X.; Bai, H.; Xue, X. Y.; Hou, Z.; Zhou, Y.; Sang, G. J.; Meng, J. R. CN 101857562, 2010 [Chem. Abstr. 2010, 153, 554743].

[36] Pastor-Navarro, N.; Garcĺa-Bover, C.; Maquieira, Á.; Puchades, R. Anal. Bioanal. Chem. 2004, 379, 1088.

[37] Mirza, S. M.; Mustafa, G.; Khan, I. U.; Zia-Ur-Rehman, M.; Shafiq, M. Acta Crystallogr., Sect. E 2010, E67, o25.

[38] Chitranshi, P.; Xue, L. Bioorg. Med. Chem. Lett. 2011, 21, 6357.

[39] Motoshima, K.; Ishikawa, M.; Hashimoto, Y.; Sugita, K. Bioorg. Med. Chem. 2011, 19, 3156

[40] Ismail, N. S. M.; Hattori, M. Bioorg. Med. Chem. 2011, 19, 374.

[41] Hutchinson, J. H.; Li, Y. W.; Arruda, J. M.; Baccei, C.; Bain, G.;
Chapman, C.; Correa, L.; Darlington, J.; King, C. D.; Lee, C.; Lorrain, D.; Prodanovich, P.; Rong, H. J.; Santini, A.; Stock, N.; Prasit, P.; Evans, J. F. J. Med. Chem. 2009, 52, 5803.

[42] Suryadevara, P. K.; Olepu, S.; Lockman, J. W.; Ohkanda, J.; Karimi, M.; Verlinde, C. L. M. J.; Kraus, J. M.; Schoepe, J.; Voorhis, W. C. V.; Hamilton, A. D.; Buckner, F. S.; Gelb, M. H. J. Med. Chem. 2009, 52, 3703.

[43] Takaya, J.; Miyashita, Y.; Kusama, H.; Iwasawa, N. Tetrahedron 2011, 67, 4455.

[44] Rewcastle, G. W.; Gamage, S. A.; Flanagan, J. U.; Frederick, R.; Denny, W. A.; Baguley, B. C.; Kestell, P.; Singh, R.; Kendall, J. D.; Marshall, E. S.; Lill, C. L.; Lee, W. J.; Kolekar, S.; Buchanan, C. M.; Jamieson, S. M. F.; Shepherd, P. R. J. Med. Chem. 2011, 54, 7105 .

[45] Brenna, E.; Fuganti, C.; Gatti, F. G.; Parmeggiani, F. Tetrahedron: Asymmetry 2009, 20, 2594.

[46] Sliman, F.; Desmaële, D. Synthesis 2010, 619.

[47] Shi, G. Q.; Dropinski, J. F.; McKeever, B. M.; Xu, S. H.; Becker, J. W.; Berger, J. P.; MacNaul, K. L.; Elbrecht, A.; Zhou, G. C.; Doebber, T. W.; Wang, P. R.; Chao, Y. S.; Forrest, M.; Heck, J. V.; Moller, D. E.; Jones, A. B. J. Med. Chem. 2005, 48, 4457.

[48] Kumar, A.; Maurya, R. A.; Sharma, S.; Ahmad, P.; Singh, A. B.; Tamrakar, A. K.; Srivastava, A. K. Bioorg. Med. Chem. 2009, 17, 5285.

[49] Yang, D. C.; Yan, J. F.; Wang, L. F.; Ye, F.; Fan, L.; Mou, X.; Li, T. J.; Liu, H. L.; Chen, W. D.; Xu, J.; Song, X. L. CN 101735286 , 2010 [Chem. Abstr. 2010, 153, 116535].

[50] Corradi, V.; Mancini, M.; Santucci, M. A.; Carlomagno, T.; Sanfelice, D.; Mori, M.; Vignaroli, G.; Falchi, F.; Manetti, F.; Radi, M.; Botta, M. Bioorg. Med. Chem. Lett. 2011, 21, 6867.

[51] Yao, Z. Y.; Yang, D. C.; Fan, L. Chin. J. Med. Chem. 2003, 13, 16 (in Chinese). (姚志勇, 杨大成, 范莉, 中国药物化学杂志, 2003, 13, 16.)

[52] Gupta, P.; Paul, S. Green Chem. 2011, 13, 2365.

[53] Rajput, A. P.; Gore, R. P. Der Pharma Chem. 2011, 3, 409.

[54] Balaskar, R. S.; Gavade, S. N.; Mane, M. S.; Shingare, M. S.; Mane, D. V. Green Chem. Lett. Rev. 2011, 4, 91.

[55] Chou, C. T.; Yellol, G. S.; Chang, W. J.; Sun, M. L.; Sun, C. M. Tetrahedron 2011, 67, 2110.

[56] Berger, J.; Moller, D. E. Annu. Rev. Med. 2002, 53, 409.

[57] Krentz, A. J.; Bailey, C. J. Drugs 2005, 65, 385.

(Li, L.; Fan, Y.) 